\title{
THE DEPENDENCE OF STAR FORMATION ACTIVITY ON STELLAR MASS SURFACE DENSITY AND SERSIC INDEX IN zCOSMOS GALAXIES AT $0.5<z<0.9$ COMPARED WITH SDSS GALAXIES AT $0.04<z<0.08^{*}$
}

C. Maier ${ }^{1}$, S. J. Lilly ${ }^{1}$, G. Zamorani ${ }^{2}$, M. ScodegGio ${ }^{3}$, F. LAmareille ${ }^{4}$, T. Contini ${ }^{4}$, M. T. Sargent ${ }^{1,5}$, C. Scarlata ${ }^{1,6}$, P. $\mathrm{Oesch}^{1}$, C. M. Carollo ${ }^{1}$, O. Le Fèvre ${ }^{7}$, A. Renzini ${ }^{8}$, J.-P. Kneib ${ }^{7}$, V. Mainieri ${ }^{9}$, S. Bardelli $^{2}$, M. Bolzonella $^{2}$, A. Bongiorno ${ }^{10}$, K. CAputi ${ }^{1}$, G. Coppa ${ }^{2}$, O. Cucciati ${ }^{11}$, S. De la Torre ${ }^{7}$, L. De Ravel ${ }^{7}$, P. Franzetti $^{3}$, B. Garilli ${ }^{3}$, A. Iovino ${ }^{11}$, P. KAmpczyK ${ }^{1}$, C. Knobel ${ }^{1}$, K. KovaČ ${ }^{1}$, J.-F. Le Borgne ${ }^{4}$, V. Le Brun ${ }^{7}$, M. Mignoli $^{2}$, R. Pello $^{4}$, Y. Peng ${ }^{1}$, E. Perez Montero ${ }^{4}$, E. Ricciardelli ${ }^{8}$, J. D. Silverman ${ }^{1}$, M. Tanaka ${ }^{9}$, L. Tasca $^{7}$, L. Tresse $^{7}$, D. Vergani $^{2}$, E. Zucca $^{2}$,

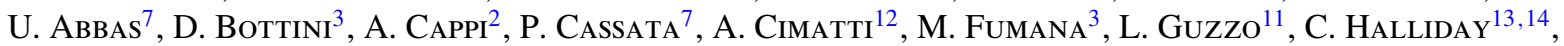
A. M. Koekemoer ${ }^{15}$, A. Leauthaud ${ }^{4}$, D. Maccagni ${ }^{3}$, C. Marinoni ${ }^{16}$, H. J. McCracken ${ }^{17}$, P. Memeo $^{3}$, B. MeneuX $^{10,18}$, C. Porciani ${ }^{1}$, L. Pozzetti $^{2}$, and R. Scaramella ${ }^{19}$

${ }^{1}$ Institute of Astronomy, ETH Zurich, CH-8093, Zurich, Switzerland; chmaier@phys. ethz.ch

2 INAF Osservatorio Astronomico di Bologna, via Ranzani 1, I-40127, Bologna, Italy

${ }^{3}$ INAF-IASF Milano, Milan, Italy

${ }^{4}$ Laboratoire d'Astrophysique de Toulouse-Tarbes, Universite de Toulouse, CNRS, 14 avenue Edouard Belin, F-31400 Toulouse, France

${ }^{5}$ Max-Planck-Institut für Astronomie, Königstuhl 17, D-69117 Heidelberg, Germany

${ }^{6}$ California Institute of Technology, MS 105-24, Pasadena, CA 91125, USA

${ }^{7}$ Laboratoire d'Astrophysique de Marseille, Marseille, France

${ }^{8}$ Dipartimento di Astronomia, Universita di Padova, Padova, Italy

${ }^{9}$ European Southern Observatory, Karl-Schwarzschild-Strasse 2, Garching, D-85748, Germany

${ }^{10}$ Max-Planck-Institut für Extraterrestrische Physik, D-84571 Garching b. Muenchen, Germany

${ }^{11}$ INAF Osservatorio Astronomico di Brera, Milan, Italy

12 Dipartimento di Astronomia, Universitá di Bologna, via Ranzani 1, I-40127, Bologna, Italy

${ }_{13}$ Osservatorio Astrofisico di Arcetri, Largo Enrico Fermi 5, 50125 Firenze, Italy

${ }^{14}$ Department of Physics and Astronomy, University of Glasgow, Glasgow G12 8QQ, UK

${ }^{15}$ Space Telescope Science Institute, 3700 San Martin Drive, Baltimore, MD 21218, USA ${ }^{16}$ Centre de Physique Theorique, Marseille, Marseille, France

${ }^{17}$ Institut d'Astrophysique de Paris, UMR 7095 CNRS, Université Pierre et Marie Curie, 98 bis Boulevard Arago, F-75014 Paris, France

${ }^{18}$ Universitäts-Sternwarte, Scheinerstrasse 1, D-81679 Muenchen, Germany

${ }^{19}$ INAF, Osservatorio di Roma, Monteporzio Catone (RM), Italy

Received 2008 July 29; accepted 2009 January 1; published 2009 March 23

\begin{abstract}
In order to try to understand the internal evolution of galaxies and relate this to the global evolution of the galaxy population, we present a comparative study of the dependence of star formation rates on the average surface mass densities $\left(\Sigma_{M}\right)$ of galaxies at $0.5<z<0.9$ and $0.04<z<0.08$, using the zCOSMOS and Sloan Digital Sky Survey (SDSS) surveys, respectively. We derive star formation rates, stellar masses, and structural parameters in a consistent way for both samples, and apply them to samples that are complete down to the same stellar mass at both redshifts. We first show that the characteristic step-function dependence of median specific star formation rate (SSFR) on $\Sigma_{M}$ in SDSS, seen by Brinchmann et al., is due to the change over from predominantly disk galaxies to predominantly spheroidal galaxies at the surface mass density $\log \Sigma_{M \text { char }} \sim 8.5$ at which the SSFR is seen to drop. Turning to zCOSMOS, we find a similar shape for the median SSFR $-\Sigma_{M}$ relation, but with median SSFR values that are about 5-6 times higher than for SDSS, across the whole range of $\Sigma_{M}$, and in galaxies with both high and low Sersic indices. This emphasizes that galaxies of all types are contributing, proportionally, to the global increase in star formation rate density in the Universe back to these redshifts. The $\Sigma_{M \text { char }}$ "step" shifts to slightly higher values of $\Sigma_{M}$ in zCOSMOS relative to SDSS, but this can be explained by a modest differential evolution in the size-mass relations of disk and spheroid galaxies. For low Sersic index galaxies, there is little change in the size-mass relation, as seen by Barden et al., although we suggest that this does not necessarily imply inside-out growth of disks, at least not in this redshift range. On the other hand, there is a modest evolution in the stellar mass-size relation for high Sersic index galaxies, with galaxies smaller by $\sim 25 \%$ at $z \sim 0.7$. Taken together these produce a modest increase in $\Sigma_{M \text { char }}$. Low Sersic index galaxies have a SSFR that is almost independent of $\Sigma_{M}$, and the same is probably also true of high Sersic index galaxies once obvious disk systems are excluded.
\end{abstract}

Key words: galaxies: evolution - galaxies: high-redshift

Online-only material: color figures

\section{INTRODUCTION}

* Based on observations obtained at the European Southern Observatory (ESO) Very Large Telescope (VLT), Paranal, Chile, as part of the Large Program 175.A-0839 (the zCOSMOS Spectroscopic Redshift Survey)
One of the key unanswered questions in the study of galaxy evolution is what physical processes inside galaxies drive the changes in the star formation rates (SFRs) in individual galaxies 
that, taken together, produce the large decline in the global star formation rate density (SFRD) to redshifts since $z \sim 2$ (e.g., Lilly et al. 1996; Hippelein et al. 2003; Hopkins \& Beacom 2006, and references therein). It is known that there is a strong correlation between SFR and stellar mass in the local Universe as shown by numerous SDSS studies (e.g., Brinchmann et al. 2004; Salim et al. 2007; Schiminovich et al. 2007). This is demonstrated, for example, in Figure 17 of Brinchmann et al. (2004), although this figure also clearly shows that at high stellar masses the distribution of SFRs broadens significantly and the correlation between stellar mass and SFR breaks down. Many studies at intermediate redshifts of the SFR or specific SFR (SSFR) have been made as a function of the integrated stellar mass of galaxies (e.g., Bauer et al. 2005; Bundy et al. 2006; Noeske et al. 2007a, 2007b) but these did not use information on the internal structural properties of the galaxies.

In contrast, studies using the local Sloan Digital Sky Survey (SDSS) sample (e.g., Brinchmann et al. 2004) have argued that the surface mass density may be more important than stellar mass in regulating star formation. Using the SDSS sample Brinchmann et al. (2004) found that the low SSFR peak is more prominent at high $\Sigma_{M}$ than at high $M_{*}$, and therefore concluded that the surface density of stars is more important than stellar mass in regulating star formation. In a follow-up study, Kauffmann et al. (2006) found that the total spread in SSFR reaches a maximum at a characteristic stellar surface mass density $\Sigma_{M \text { char }}$, and interpreted this as a qualitative change in the distribution of star formation histories above and below $\Sigma_{M \text { char }}$. The behavior of the SSFR with $\Sigma_{M}$ follows a smoothed "step-function" dropping substantially at a characteristic $\Sigma_{M \text { char }}$.

This observation and the resulting discussion therefore motivates the current investigation. Using the HST/ACS images of the COSMOS field, plus star formation rate information from emission lines measured in large numbers of zCOSMOS spectra we can study the changes that have occurred in the SSFR $-\Sigma_{M}$ relation between redshifts approaching $z \sim 1$ and the present epoch, as sampled by the SDSS studies, provided we can select comparable samples at the different redshifts, e.g., above a certain integrated stellar mass.

The goal of this paper is therefore to obtain clues about the links between the internal evolution of galaxies, in particular the build-up of stellar mass, and the global changes that are seen in the population of galaxies as a whole, by studying this SSFR$\Sigma_{M}$ relationship at significantly earlier epochs, $0.5<z<0.9$.

This work represents an improvement over an earlier study of the SSFR versus stellar mass surface density in the COSMOS field by Zamojski et al. (2007) by using secure spectroscopic redshifts, by deriving SFRs from emission line fluxes rather than ultraviolet continuum luminosities, by carefully constructing compatible mass-complete subsamples for both zCOSMOS and SDSS, by computing half-light radii in a consistent way, at the same rest-frame wavelength, and by exploring the contributions of galaxies with different Sersic indices to the overall SSFR $-\Sigma_{M}$ relation.

By focusing on internal properties of galaxies, this paper complements a number of other studies of the evolution of galaxies over the $0<z<1$ redshift range that are being carried out using the first 10,000 spectra from the zCOSMOS redshift survey (e.g., Caputi et al. 2008, 2009; Mignoli et al. 2009; Silverman et al. 2008). These other papers are more focused on the integrated properties of galaxies and on the variation of these with the external galaxian environment.

This paper is organized as follows: Section 2 describes our sample selection and the derivation of the relevant physical quantities for both zCOSMOS $(0.5<z<0.9)$ and SDSS $(0.04<z<0.08)$ galaxies. In Section 3, we present our main results regarding the role of stellar mass and stellar mass surface density in regulating star formation activity for zCOSMOS and SDSS galaxies as a function of morphology. These include new insights into the form of the SSFR $-\Sigma_{M}$ relation at the present epoch, and a comparison of the changes seen in this relation back to $0.5<z<0.9$. These results are discussed in Section 3, and conclusions are presented in the last section. A cosmology with $H_{0}=70 \mathrm{~km} \mathrm{~s}^{-1} \mathrm{Mpc}^{-1}, \Omega_{0}=0.25, \Omega_{\Lambda}=0.75$ is used throughout this paper.

\section{DATA SETS}

\subsection{The zCOSMOS Sample at $0.5<z<0.9$}

The Cosmic Evolution Survey (COSMOS; Koekemoer et al. 2007; Scoville et al. 2007a) is the largest Hubble Space Telescope (HST) survey ever undertaken, imaging an equatorial $1.64 \mathrm{deg}^{2}$ field with single-orbit $I$-band exposures (Scoville et al. 2007b). The zCOSMOS project (Lilly et al. 2007) is securing spectroscopic redshifts for large numbers of galaxies in the COSMOS field. We now have in hand spectra of about 10,500 $I_{A B} \leqslant 22.5 I$-band selected galaxies over $1.5 \mathrm{deg}^{2}$ of the COSMOS field, the so-called zCOSMOS-bright $10 \mathrm{k}$ sample (S. J. Lilly et al. 2009, in preparation). The zCOSMOS-bright sample is a flux-limited sample of galaxies with $I_{A B}<22.5$ generated from the COSMOS HST ACS images. The sample does not have a significant observational surface brightness selection, but, as with all samples selected on total magnitude, there is a selection in surface brightness at a given size. The success rate of measuring redshifts varies with redshift and is very high (more than 90\%) between $0.5<z<0.9$. For more details about the zCOSMOS survey, we refer the reader to Lilly et al. (2007) and S. J. Lilly et al. (2009, in preparation).

Spectroscopic observations in zCOSMOS-bright were acquired using VIMOS with the $R \sim 600 \mathrm{MR}$ grism over a spectral range over 5550-9650 $\AA$. This enables us to study rest-frame spectral features around $4000 \AA$, such as the [O II] $\lambda 3727$ emission line, to $z \sim 1.2$.

\subsubsection{Sample Selection}

Only 10k sample galaxies with reliable redshifts are used for this study. Specifically, and with reference to the scheme described by S. J. Lilly et al. (2009, in preparation), we use redshifts of confidence classes $4,3,2.5,2.4,9.5,9.4,9.3$, and 1.5. These have an overall reliability, based on repeat observations and on overall consistency with independent photometric redshifts, of more than $99 \%$.

We exclude about 500 stars, the broad line active galactic nuclei (AGNs), and the few galaxies that lie outside the ACS fields, leaving us with a sample of 8131 galaxies at $z<1.2$ for which structural parameters are available from the HST/ACS images from GIM2D Sersic fits, as described in detail by Sargent et al. (2007). Automated morphological classifications using the ZEST scheme (ZEST; Scarlata et al. 2007) are also available.

To have a high degree of spectroscopic completeness (see Figure 9 in Lilly et al. 2007) and for additional reasons discussed below, we further restrict the zCOSMOS sample for this study to galaxies lying between $0.517<z<0.900$.

To avoid night sky lines problems with our chosen tracer for star formation, the [O II] $\lambda 3727$ emission line, we exclude all galaxies for which the center of the $[\mathrm{O}$ II] $\lambda 3727$ line is less 
than $12 \AA$ away from the center of a strong night sky line. We therefore exclude galaxies with $\mathrm{O}$ II lines close to the strong night sky lines $5893 \AA$, [O I] $6300 \AA$, $6364 \AA$, or $6832 \AA$, i.e., at redshifts $0.5771<z<0.5852,0.6871<z<0.6936$, $0.7043<z<0.7108$, and $0.8299<z<0.8363$, respectively. We exclude galaxies in the redshift range $z<0.5174$ to avoid problems with the $[\mathrm{O}$ II] line flux measurements using the automatic software Platefit_VIMOS (F. Lamareille et al. 2009, in preparation) at the blue edge of the spectrum. This way we remain with 3232 galaxies.

\subsubsection{Line Measurements and AGN Rejection}

Emission line fluxes were measured using the automatic routine Platefit_VIMOS (F. Lamareille et al. 2009, in preparation). After removing a stellar component using Bruzual \& Charlot (2003) models, Platefit_VIMOS completes a simultaneous Gaussian fit of all emission lines using a Gaussian profile. If an emission line measurement has a significance of less than $1.15 \sigma$ (this value is derived using the distribution of fake detections, as described in F. Lamareille et al. 2009, in preparation), then an upper limit is calculated for the respective line.

For galaxies for which Platefit_VIMOS detects all three emission lines [O III] $\lambda 5007, \mathrm{H} \beta$, and [O II] $\lambda 3727$, we use the $[\mathrm{O}$ III $] \lambda 5007 / \mathrm{H} \beta$ versus [O II] $\lambda 3727 / \mathrm{H} \beta$ diagram to distinguish star formation dominated galaxies from objects obviously containing an active nucleus (narrow-line AGNs) with the aid of the empirical threshold derived using the 2dFGRS by Lamareille et al. (2004). Additionally, all the X-ray detected AGN (Brusa et al. 2007) are excluded, regardless of their spectral properties.

This results in a sample of 3048 galaxies at $0.5<z<0.9$, comprising 1527 galaxies at $0.5<z<0.7$, and 1521 galaxies at $0.7<z<0.9$. These objects are used for the analysis below.

\subsubsection{Structural Parameters}

Physical sizes are computed from the half-light radii, $r_{1 / 2}$, defined to be the semimajor axis of the ellipse containing half of the total flux, derived from the GIM2D Sersic fits of Sargent et al. (2007). These GIM2D fits also provide Sersic indices. It should be noted that GIM2D provides a surface brightness profile for each galaxy as it would be in the absence of the instrumental point-spread function (PSF).

As described in Sargent et al. (2007), extensive tests and simulations showed that these fits slightly underestimate the half-light radii of galaxies. Therefore, a correction was applied to the half-light radii, following their Figure 21, which depends on the half-light radius size in pixels, the $I$-band magnitude (i.e., the signal to noise), and the Sersic index of the galaxy in question. This correction was $\leqslant 10 \%$ for $78 \%$ of the 3048 zCOSMOS galaxies at $0.5<z<0.9$, and more than $20 \%$ for just $2 \%$ of the sample.

Although the ZEST automated morphological classification (Scarlata et al. 2007) is available for the entire zCOSMOS sample, it has not been applied to the SDSS. Therefore, for consistency, we decided to simply use Sersic indices $(n)$ to compare the structures (morphologies) of zCOSMOS and SDSS galaxies. As is well known (e.g., Blanton et al. 2003a; Scarlata et al. 2007) $n>2.5$ galaxies correspond mostly to galaxies classified as early-types spheroid-dominated galaxies, while $n<1.5$ galaxies are mostly objects classified as late-type diskdominated galaxies.
In what follows, we will split the samples according to Sersic index, and loosely refer to these as disk- and spheroid-dominated galaxies, as late and early type, etc., while endeavoring to keep the Sersic criteria clear.

\subsubsection{Ellipticity}

In addition to the Sersic indices, we also use an ellipticity $(q=1-b / a)$ criterion to separate galaxies. There are two motivations for this:

First, Maller et al. (2009) have shown that $90 \%$ of galaxies with an axis ratio $b / a \leqslant 0.55$ are disk galaxies in an SDSS sample. Therefore, eliminating high Sersic galaxies that nevertheless have a low $b / a$ ratio should produce a cleaner sample of early-type galaxies, since these highly inclined galaxies are unlikely to be true early types. Secondly, the low Sersic index disk galaxies with $b / a \leqslant 0.55$ are likely to be more affected by dust extinction (see e.g., Möllenhoff et al. 2006), affecting estimates of stellar masses based on the spectral energy distributions (SEDs), SFRs derived from emission lines and possibly also their size estimates, all of which become more difficult. Eliminating highly inclined galaxies with low Sersic indices also therefore has advantages.

For both these reasons, we will often focus on the subset of galaxies with $b / a>0.55$ in both the zCOSMOS and SDSS samples, and will do so regardless of their Sersic index. By doing so, we obtain a cleaner sample of early types and eliminate the hardest to interpret late types. Applied consistently between the SDSS and high redshift samples, neither should introduce systematic biases.

\subsubsection{Stellar Masses and Surface Mass Density}

Stellar masses, and the surface mass densities, must be derived from estimating the mass-to-light ratio (M/L) of the stellar population using multiband photometry. The advantages of doing this using the widest possible wavelength range and extending as far as possible into the rest-frame near-infrared are well known.

Unfortunately, while such data exist in the COSMOS field, systematic near-infrared photometry is not yet available for SDSS. Therefore, in order to achieve the highest possible internal consistency for our study, we therefore derive mass estimates from purely rest-frame optical colors, for both SDSS and zCOSMOS, using the equation given below in Equation (1).

We have however compared these stellar masses with masses derived using the entire COSMOS optical to infrared SED and Bruzual \& Charlot (2003) models by M. Bolzonella et al. (2009, in preparation). As shown in Figure 1 these show good agreement with a statistical rms of around 0.13 dex per galaxy and an offset (to higher SED masses) of $0.10 \mathrm{dex}$ in the mean, reflecting typical uncertainties in derived stellar masses. Interestingly, as described by M. Bolzonella et al. (2009, in preparation), population synthesis models with TP-AGB phase (Maraston 2005) would produce a systematic shift of $\sim 0.1 \mathrm{dex}$ toward lower SED masses than those shown in Figure 1, thus eliminating the offset between the masses computed using Equation (1) and the SED masses.

Stellar masses are derived using the relation between restframe $U-B$ and $B-V$ colors and $\mathrm{M} / \mathrm{L}$, using Equation (1) from Lin et al. (2007), which corrects the $\mathrm{M} / \mathrm{L}$ for evolution, and accounts for variations in the $\mathrm{M} / \mathrm{L}$ with color. The relation from Lin et al. (2007) is modified as follows, and applied to both 


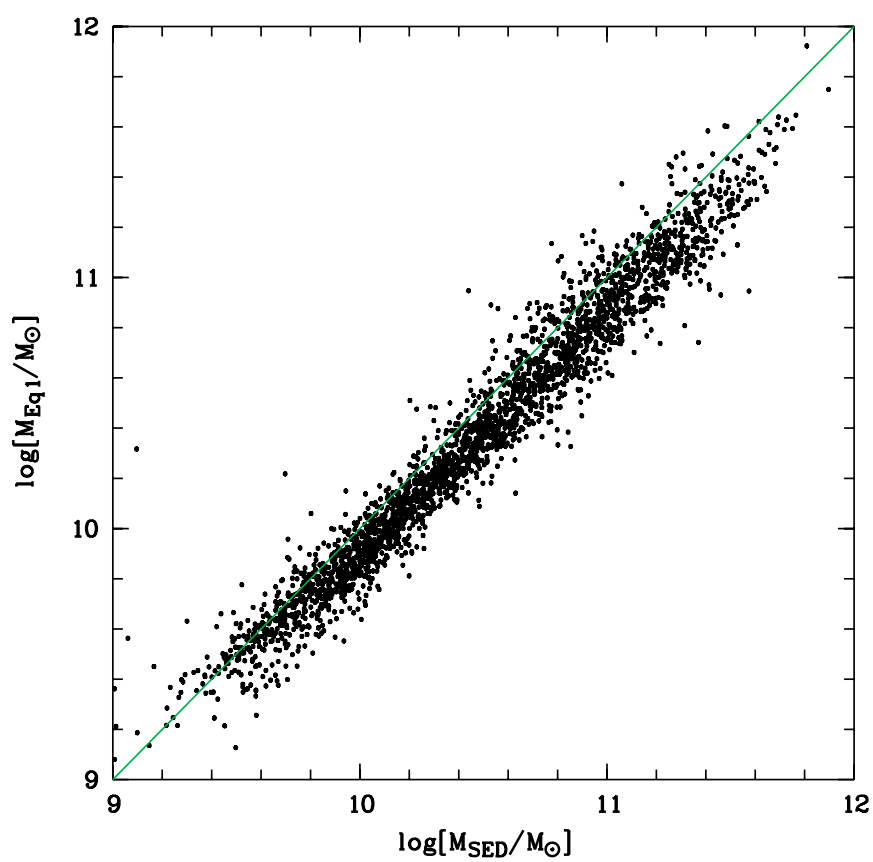

Figure 1. Comparison of the stellar masses computed using Equation (1) with masses derived using the entire COSMOS optical to infrared SED and Bruzual \& Charlot (2003) models by M. Bolzonella et al. (2009, in preparation). A good agreement with a statistical rms of around 0.13 dex per galaxy and an offset (to higher SED masses) of $0.10 \mathrm{dex}$ in the mean is seen, reflecting typical uncertainties in derived stellar masses. Interestingly, as described by M. Bolzonella et al. (2009, in preparation), population synthesis models with TP-AGB phase (Maraston 2005) would produce a systematic shift of $\sim 0.1 \mathrm{dex}$ towards lower SED masses than those shown in the figure, thus eliminating the offset between the masses computed using Equation (1) and the SED masses. In order to achieve the highest possible internal consistency for our study, we derive mass estimates from purely rest-frame optical colors, for both SDSS and zCOSMOS, using Equation (1) (see discussion in Section 2.1.5)

(A color version of this figure is available in the online journal.)
SDSS and zCOSMOS data sets:

$$
\begin{aligned}
& \log \left(M_{*} / M_{\odot}\right)=0.4 \cdot\left(5.48-M_{B}-0.10\right) \\
& \quad+1.737 \cdot\left(M_{B}-M_{V}+0.1\right)+0.098 \cdot\left(M_{U}-M_{B}-0.81\right) \\
& \quad-0.130 \cdot\left(M_{U}-M_{B}-0.81\right)^{2}-0.268 \cdot z-1.003 \\
& \quad+\log (1.7) .
\end{aligned}
$$

The difference between Equation (1) in Lin et al. (2007), which uses Vega magnitudes, and our Equation (1), is due to the transformation onto the $\mathrm{AB}$ system, using the conversions given in Table 1 in Willmer et al. (2006). Finally, since our estimate of the SFR from Moustakas et al. (2006) is based on a Salpeter initial mass function (IMF; Salpeter 1955), and the stellar masses computed by Lin et al. (2007) assume a Chabrier IMF (Chabrier 2003), we convert the latter masses to a Salpeter IMF statistically, using the conversion factor of 1.7 (Pozzetti et al. 2007). This accounts for the last term in Equation (1).

For zCOSMOS galaxies, $M_{U}, M_{B}$, and $M_{V}$ are Johnson rest-frame absolute magnitudes in the $\mathrm{AB}$ system and were calculated from the best-fitting continuum template for each galaxy. This was obtained by fitting the multiwavelength COSMOS SED that is available for each object (Capak et al. 2007; Taniguchi et al. 2007), using the ZEBRA photo-z code (Feldmann et al. 2006) at the known, spectroscopic, redshift. To achieve internal consistency with the size measurements, the SEDs were previously normalized to the actual (half-light) $I$-band magnitude measured from the GIM2D fits.

Finally, surface mass densities were computed using $\log \Sigma_{M} /\left[M_{\odot} \mathrm{kpc}^{-2}\right]=\log \left(M_{*} / M_{\odot}\right)-\log \left[2 \pi\left(r_{1 / 2} / \mathrm{kpc}\right)^{2}\right]$, for both the zCOSMOS and SDSS galaxies. Further details on the calculation of half-light radii for the SDSS sample are given in Section 2.2. Since many studies of galaxy evolution at $z<1$ are based on stellar masses, in Figure 2 we show, for the benefit of the reader, the comparison between $\Sigma_{M}$ and stellar mass for the

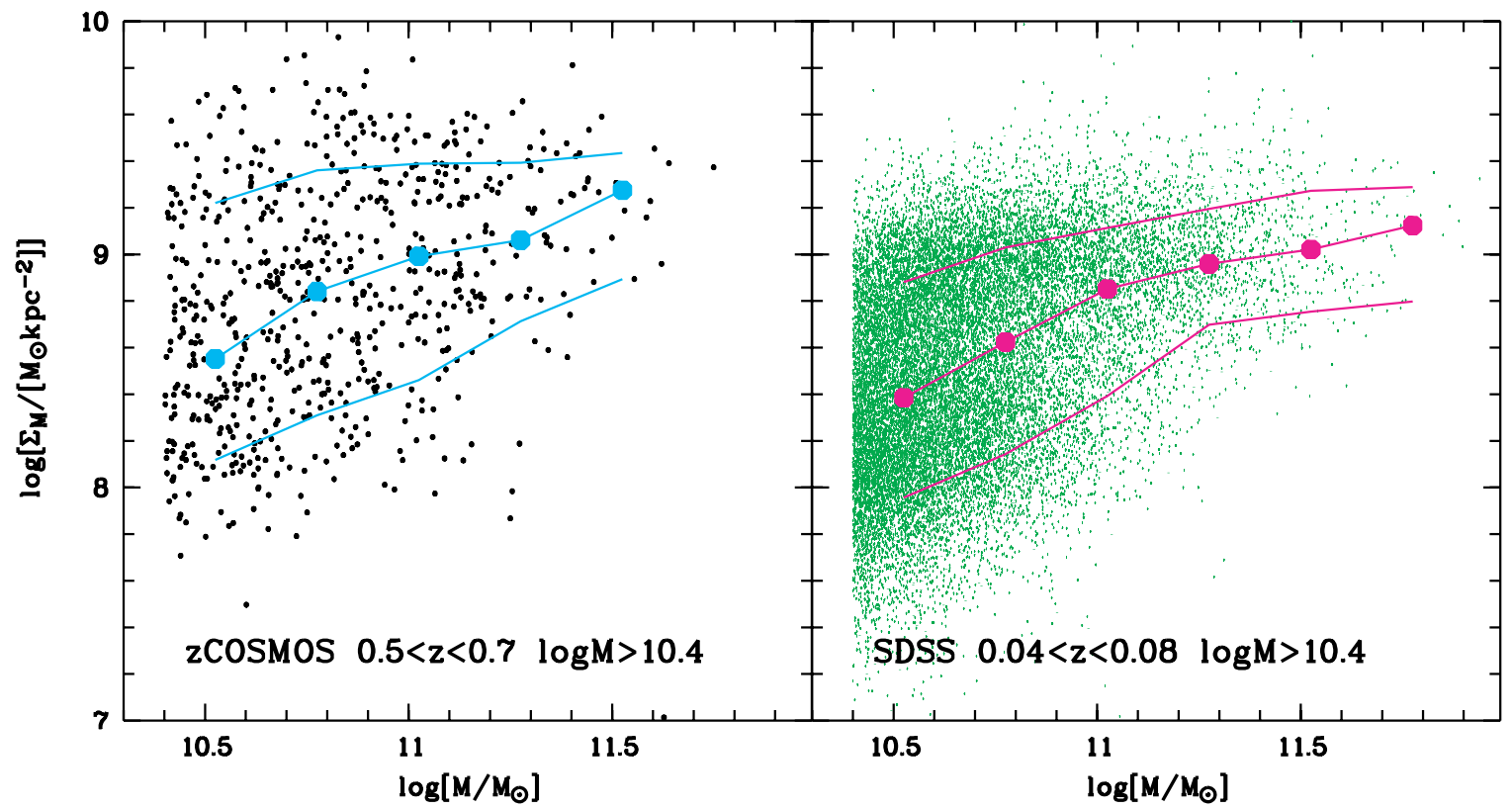

Figure 2. Comparison between stellar mass surface density $\Sigma_{M}$ and stellar mass for zCOSMOS and SDSS mass-complete samples (see Figure 4). The median $\Sigma_{M}$ values in different stellar mass bins are shown as filled circles, and 16th and 84th percentiles as solid lines. A slight increase of $\Sigma_{M}$ with stellar mass, but with a large scatter, can be seen for both zCOSMOS and SDSS samples of relatively massive galaxies (see Section 2.1.5).

(A color version of this figure is available in the online journal.) 

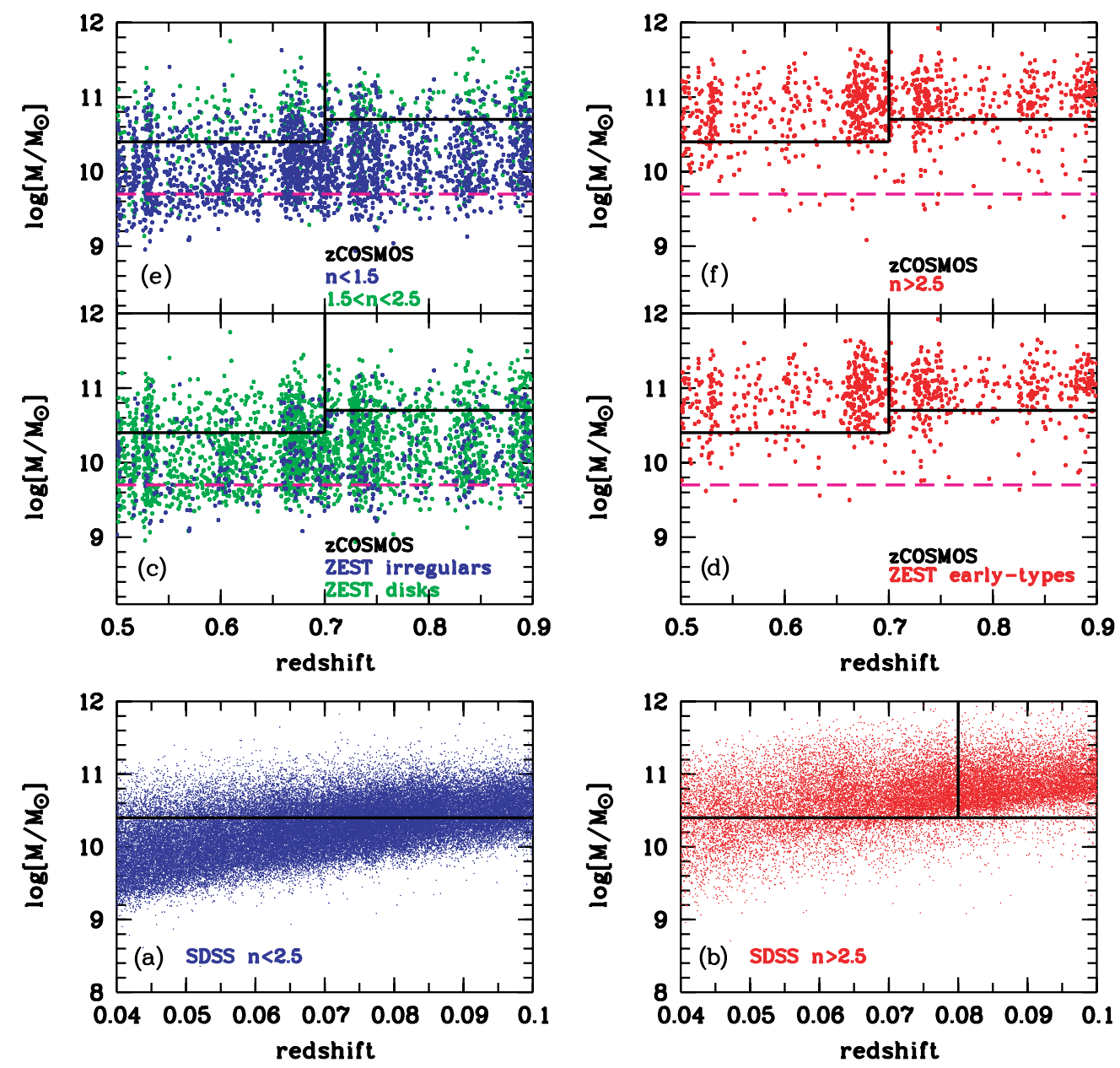

Figure 3. The stellar mass as a function of redshift for the SDSS sample (panels (a) and (b)), and for zCOSMOS galaxies at $0.5<z<0.9$ (panels (c)-(f)) for different Sersic indices, $n$. The analysis in this paper is based on the mass-complete samples with $\log M_{*}>10.4$ and $\log M_{*}>10.7$ for $0.5<z<0.7$ and $0.7<z<0.9$. In order to be complete for SDSS galaxies (panel (b)) for the same mass range $\left(\log M_{*}>10.4\right)$ as for zCOSMOS galaxies, a redshift range of $0.04<z<0.08$ for the comparison SDSS sample was chosen.

(A color version of this figure is available in the online journal.)

zCOSMOS and SDSS mass-complete samples (see below the discussion of the mass completeness). A slight increase of $\Sigma_{M}$ with stellar mass, but with a large scatter, can be seen for both zCOSMOS and SDSS samples of relatively massive galaxies.

\subsubsection{Star Formation Rates}

One of the most reliable and well calibrated SFR indicators is the $\mathrm{H} \alpha$ emission line, but the drawback for many high redshift studies is that $\mathrm{H} \alpha$ is redshifted out of the optical window beyond $z \sim 0.5$, and its measurement requires very time-consuming near-infrared spectroscopy for small samples of galaxies (see, e.g., Maier et al. 2005, 2006). Therefore, the strongest emission feature in the blue, the [O II] forbidden-line doublet, is usually used at $z>0.5$ as a tracer of the SFR. We use the [O II] $\lambda 3727$ line to SFR calibration of Moustakas et al. (2006) for this study, for both the zCOSMOS objects at $0.5<z<0.9$ and the SDSS galaxies at $0.04<z<0.08$. The two dominant sources of scatter in optical SFR diagnostics, dust extinction and metallicity, both correlate strongly with $L(B)$ (see e.g., Figure 16 in Moustakas et al. 2006), and this motivated their empirical calibration of SFR in terms of the [O II] luminosity. This calibration was developed by tuning [O II]derived SFRs to match those based on extinction-corrected $\mathrm{H} \alpha$ using observations of nearby galaxies. It might be a concern that the extinction changes at intermediate redshift, but our own observations of $\mathrm{H} \alpha$ in a limited sample of CFRS galaxies at $0.5<z<0.9$ (Maier et al. 2005) indicate that neither average extinction nor average metallicity have greatly changed for most galaxies at this redshift. Moreover, Moustakas et al. (2006) showed their calibration to be effective also at intermediate redshifts.

In detail, the conversion of [O II] luminosity to SFR is undertaken as follows. First, because the slits in the VIMOS masks have a width of 1 arcsec, an aperture correction to the [O II] line flux is applied before computing the SFRs. Each zCOSMOS spectrum is convolved with the ACS I(814) filter and then this magnitude is compared with the I-band magnitude of the GIM2D fits of Sargent et al. (2007). The difference between the two magnitudes gives the aperture correction factor for each spectrum. For more than $85 \%$ of the sample, these aperture correction factors are between 1 and 3 . This correction assumes that the $[\mathrm{O} \mathrm{II}]$ line flux and $I$-band continuum suffer equal slit losses.

Then, the corrected [O II] line luminosities are transformed into SFRs using a correction factor based on the galaxy's $B$-band absolute magnitude, as given by a linear interpolation of the $\langle R\rangle$ values in Table 2 of Moustakas et al. (2006), and shown in their Figure 19. We use the following formula to convert the [O $\mathrm{II}]$ 


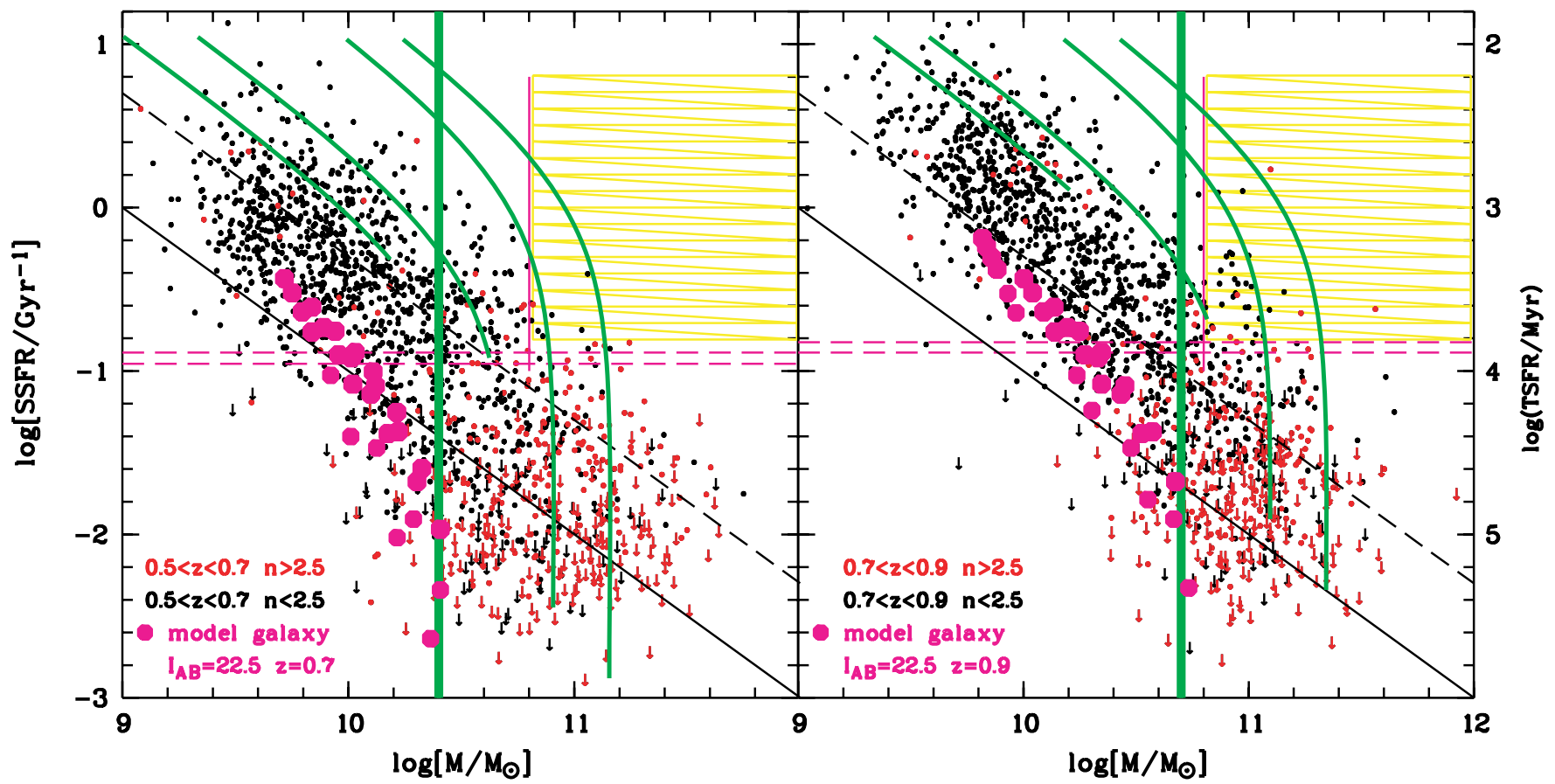

Figure 4. SSFR vs. stellar mass as a function of Sersic indices for the 3048 zCOSMOS galaxies at $0.5<z<0.9$ selected as described in Sections 2.1.1 and 2.1.2. The solid and dashed diagonal lines in each panel show SFRs of 1 and $5 M_{\odot} / y$ r, respectively. The solid green lines show the paths of a model galaxy to redshift $z \sim 0.6$ (left panel) and $z \sim 0.8$ (right panel) with different redshifts $z_{f}$ of the assumed onset of the star formation, and different e-folding timescale of the star formation $\tau$ : $z_{f}=1$ and $\tau=5 \mathrm{Gyr}, z_{f}=2$ and $\tau=3 \mathrm{Gyr}, z_{f}=3$ and $\tau=1 \mathrm{Gyr}$, and $z_{f}=5$ and $\tau=1 \mathrm{Gyr}$, respectively (from left to right). The large magenta filled circles show the location of a set of models normalised to have $I_{A B}=22.5$ (the zCOSMOS selection limit) at $z=0.7$ (left panel), and $z=0.9$ (right panel), produced using Bruzual \& Charlot (2003) models with a wide range of different star formation histories. These differ in having different SFR e-folding timescales ( $\tau$ from $5 \mathrm{Gyr}$ to $0.7 \mathrm{Gyr}$ ), and different starting redshifts ( $z_{f}$ from 1.3 to 5 ). The magenta filled circles effectively indicate the completeness limit of the sample, in the sense that the sample misses galaxies that lie to the left of these. The adopted mass completeness limits shown by the green vertical thick lines are $\log M_{*}>10.4$ for $0.5<z<0.7$, and at $\log M_{*}>10.7$ for $0.7<z<0.9$. This figure shows two well known phenomena: first, the well known downsizing effect (illustrated by the much higher number of galaxies in the yellow hatched region at $0.7<z<0.9$ compared to $0.5<z<0.7$ ), and secondly, the fact that galaxies with different Sersic indices have different star formation histories, even at a fixed mass (see discussion in Section 3.1).

luminosity into SFR assuming a Salpeter IMF (Salpeter 1955) for both luminous SDSS and zCOSMOS galaxies:

$$
\begin{aligned}
\log \left[\mathrm{SFR} / M_{\odot} \mathrm{yr}^{-1}\right]= & \log \left[L_{[\mathrm{O}} \text { II] } /\left(\mathrm{ergs} \mathrm{s}^{-1}\right)\right] \\
& -41-0.195 \cdot M_{B}-3.434 .
\end{aligned}
$$

We have checked that the SFRs derived using Equation (2) are in broad agreement with those derived by L. Pozzetti et al. (2009, in preparation) using the entire COSMOS optical to infrared (including $K, 3.6 \mu \mathrm{m}$, and $4.6 \mu \mathrm{m}$ ) SED.

\subsubsection{Mass Completeness of the zCOSMOS Sample}

Both zCOSMOS and SDSS are flux-limited samples. Since the $\mathrm{M} / \mathrm{L}$ will depend on the stellar population, which itself will produce different SEDs and rest-frame colors, both the SDSS and zCOSMOS samples will have a complicated massselection function. The problem is more acute for zCOSMOS because we can choose the SDSS redshift range so that the SDSS sample is effectively complete for the masses of interest. Of course, working in terms of stellar mass instead of luminosity is useful not only because it is closer to quantities predicted by theory, but also because it in principle removes the evolution in luminosity due to the aging of stellar populations. Blue late-type star-forming galaxies generally have lower M/L. Therefore, in a given flux-limited survey such as zCOSMOS, they are detected to smaller masses than are red early-type galaxies, simply because the latter are fainter at a given mass, i.e., have a higher M/L. This is shown in Figure 3 (stellar mass versus redshift) and Figure 4 (SSFR versus stellar mass diagram).
For this reason, if we calculate the maximum $\mathrm{M} / \mathrm{L}$ at a given redshift, i.e., that of a red passively evolving galaxy, and apply this to calculate the minimum mass that a galaxy must have in order to be brighter than our flux limit, then we can safely assume that all other galaxies with masses above this mass limit will be visible within the survey, because they will have lower M/Ls.

We have explored this by running a wide range of Bruzual \& Charlot (2003) models, with different SFR e-folding timescales ( $\tau$ from $5 \mathrm{Gyr}$ to $0.7 \mathrm{Gyr}$ ), and with different starting redshifts $\left(z_{f}\right.$ from 1.3 to 5$)$. For each model, we scale the model so that it has an observed $I_{\mathrm{AB}}=22.5$ (the flux density limit of zCOSMOS) at either $z=0.7$ or $z=0.9$, and plot the mass and SSFR of this "just-detectable" model on Figure 4 as large magenta dots. While there is scatter because of the range of models used, these magenta dots give a good indication of the mass completeness limit of the sample: galaxies that occupy the diagram to the left of the filled circles will not be seen while those to the right will have been detected.

As the $\mathrm{M} / \mathrm{L}$ asymptotes to a limiting value the locus of the dots becomes vertical and this limit is adopted as the overall mass completeness limit. These adopted mass completeness limits are shown by the green solid vertical thick lines in the two panels of Figure 4.

Henceforth, we only consider objects above these mass limits for the two zCOSMOS redshift ranges. At $\log M_{*}>10.4$, we obtain a mass-complete $\mathrm{zCOSMOS}$ sample of 648 galaxies at $0.5<z<0.7$, while for $\log M_{*}>10.7$ we obtain a masscomplete zCOSMOS sample of 520 galaxies at $0.7<z<0.9$. 


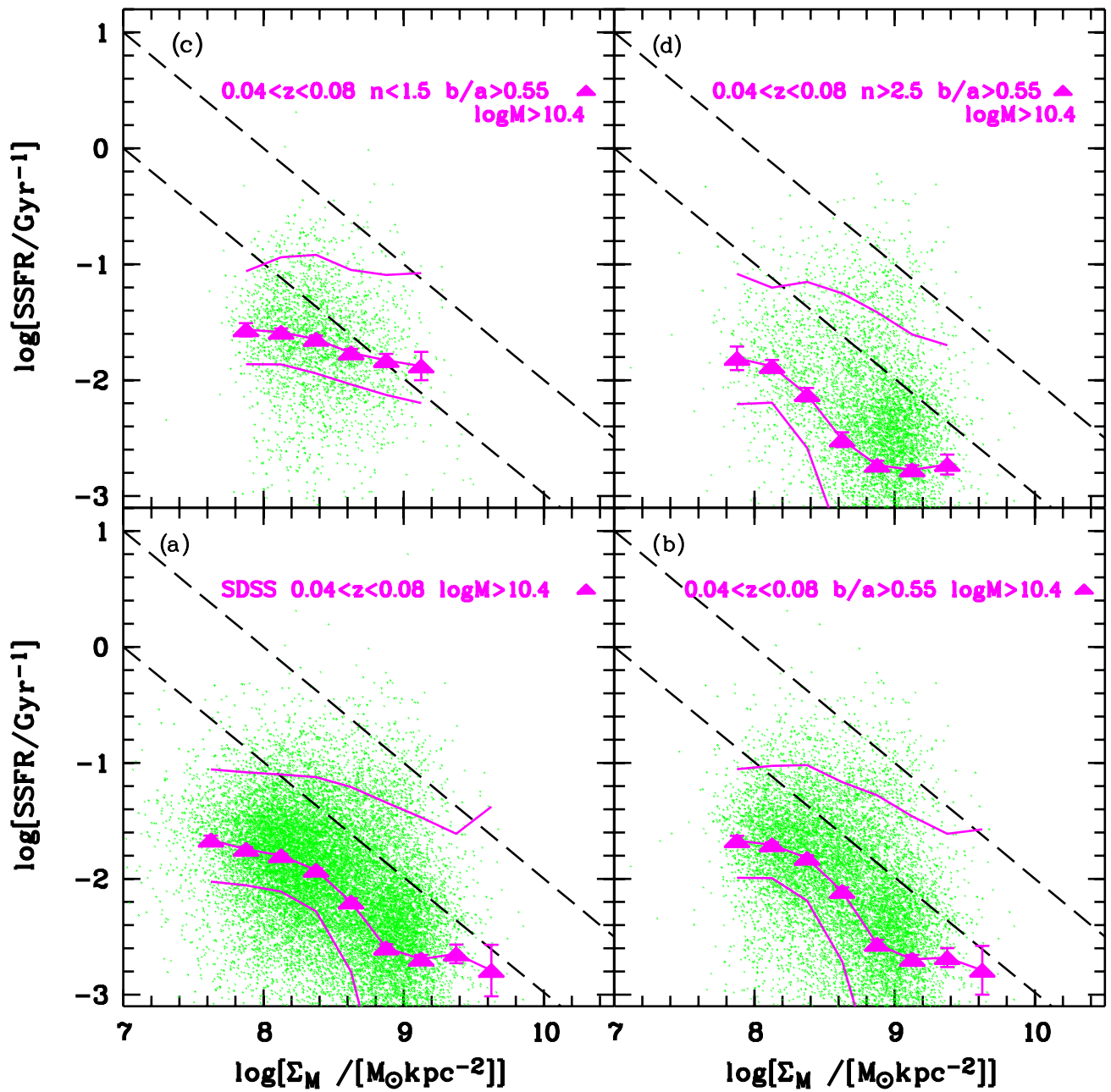

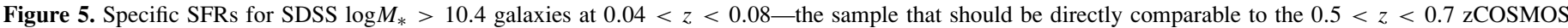

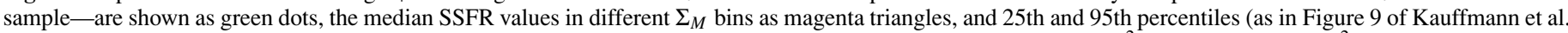

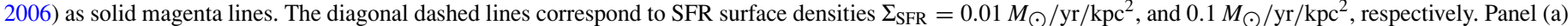

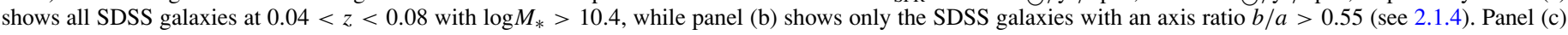

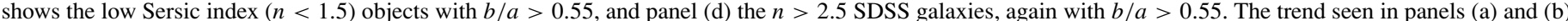

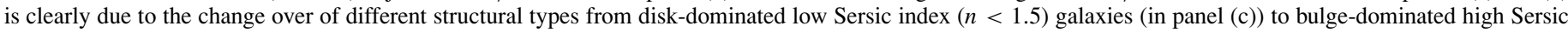
$(n>2.5)$ index galaxies (panel (d)) as the $\Sigma_{M}$ increases. This change over is also clearly seen in Figure 6.

(A color version of this figure is available in the online journal.)

As noted above and described below, the SDSS redshift range is chosen so that the SDSS objects are intrinsically faint enough that we can obtain the equivalent mass-complete samples also in SDSS. In what follows, we will always compare zCOSMOS and SDSS samples selected to the same mass limits. It should be noted that these mass limits are determined at the epoch at which the galaxy is observed, and do not, therefore account for any stellar mass added in between. As we remark below, we would expect this added mass to be modest $(0.1 \mathrm{dex})$ in most cases.

\subsection{The SDSS Comparison Sample}

We have selected a local comparison sample of SDSS galaxies from the DR4 release, Garching repository (http://www.mpagarching.mpg.de/SDSS/DR4/), in the redshift range $0.04<$ $z<0.08$. The lower redshift limit of $z=0.04$ is chosen following the recommendation of Kewley et al. (2005) to reduce the systematic and random errors in the SFRs that arise from aperture effects due to the 3 arcsec size of the SDSS fibers. The upper limit choice of $z=0.08$ ensures mass completeness of red (high M/L.) SDSS galaxies at $\log M_{*}>10.4$, as shown in panel (b) in Figure 3. We selected galaxies with Petrosian $r$ magnitudes in the range $14.5<r<17.77$. The bright limit is necessary because SDSS becomes incomplete for bright galaxies of large angular size and to avoid objects with saturated SDSS photometry, whereas the faint limit corresponds to the nominal magnitude limit of the main galaxy sample in SDSS. It should be noted that duplicate objects, and SDSS galaxies on problematic or special plates are excluded from the SDSS sample. This exclusion is based solely on position on the sky and should not be related to any galaxy properties.

To ensure a consistent comparison of the physical properties of the SDSS and zCOSMOS samples, we perform the following steps to derive stellar masses, sizes, Sersic indices, and SFRs for SDSS galaxies.

1. To derive the sizes $r_{e 1 / 2 g}$ of SDSS galaxies, we start from the Petrosian radius containing $50 \%$ of the Petrosian flux in the $g$ band, rpet $_{50 g}$. The observed $g$ band for SDSS galaxies at $0.04<z<0.08$ corresponds approximately to rest-frame $B$ band (to be precise, effective wavelengths 


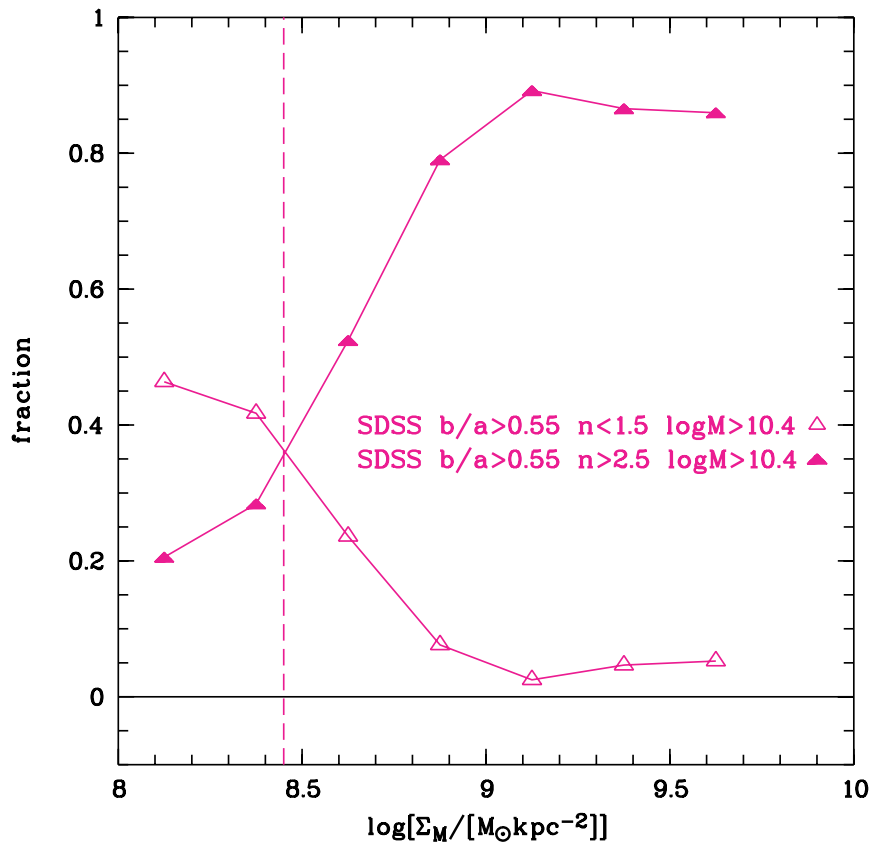

Figure 6. The fraction of SDSS objects (with $b / a>0.55$ ) with $n<1.5$ (open magenta triangles) and with $n>2.5$ (filled magenta triangles) in the $\log M_{*}>10.4$ mass-complete sample. The fraction of $n>2.5$ SDSS objects shows a sharp increase at $\log \Sigma_{M \text { trans }} \sim 8.45$, very close to the characteristic surface mass density $\log \Sigma_{M \text { char }} \sim 8.5$ found by Kauffmann et al. (2006).

(A color version of this figure is available in the online journal.)

between 4587 and $4417 \AA$ ) and is therefore consistent with the derivation of sizes for the zCOSMOS sample from the observed ACS I-band images, which have effective wavelengths of $5373 \AA$ at $z=0.5,4741 \AA$ at $z=0.7$, and $4242 \AA$ at $z=0.9$. Since these are rather similar, and always above the $4000 \AA$ break, any dependence on redshift (the so-called morphological $k$-correction) should be small. We transform rpet $_{50 g}$ into half-light radii using Equation (6) of Graham et al. (2005): $r_{1 / 2 g}=\operatorname{rpet}_{50 g} /(1-$ $\left.6 \times 10^{-6}\left(\text { rpet }_{90 \mathrm{~g}} / \text { rpet }_{50 \mathrm{~g}}\right)^{8.92}\right)$, where rpet $_{90 \mathrm{~g}}$ is the Petrosian radius containing $90 \%$ of the Petrosian flux. This equation uses the concentration rpet $_{90 \mathrm{~g}} / \mathrm{rpet}_{50 \mathrm{~g}}$ to correct for the light outside the Petrosian aperture: this flux deficit is $0.20 \mathrm{mag}$ in the case of de Vaucouleurs $R^{1 / 4}$ profile, and 0.50 mag for an $R^{1 / 8}$ profile. Then, to be consistent with the zCOSMOS $r_{1 / 2}$ measurements and since the SDSS Petrosian flux was measured with a circular aperture, we transform $r_{1 / 2 g}$ to $r_{e 1 / 2 g}$, the semimajor axis of the ellipse containing half of the total flux: $r_{e 1 / 2 g}=r_{1 / 2 g} \times \sqrt{a / b}$, where $a / b$ is the ratio between the major and minor axis of the ellipse. We did several tests to assure the consistency of the derivation of sizes for the zCOSMOS and SDSS samples, and the results are presented in Appendix A. This $r_{e 1 / 2 g}$ value, the SDSS half-light radius shown in the following in different diagrams, is then used to derive surface mass densities as described in Section 2.1.5.

2. For the reason explained above in Section 2.1.5, we use Equation (1) to derive stellar masses for the SDSS galaxies. SDSS $u, g$, and $r$ (Petrosian) magnitudes have been $k$-corrected to $z=0$ according to Blanton et al. (2003b), using $k$-correct version 4.1 .4 which is available at http://cosmo.nyu.edu/blanton/kcorrect/. $U_{-}, B-$, and $V$-band rest-frame $\mathrm{AB}$ magnitudes were computed from these $k$-corrected $u, g$, and $r$ magnitudes, using the relations from Table 2 in Blanton \& Roweis (2007), and stellar masses were calculated using these values in Equation (1). The stellar masses obtained in this way were found to be in good agreement with the SDSS total stellar masses estimated from the SDSS spectra by Kauffmann et al. (2003a). The comparison shows a statistical rms of around $0.16 \mathrm{dex}$ per galaxy and an offset of 0.03 dex in the mean. This is comparable to the quoted uncertainties in the spectral measurements (Kauffmann et al. 2003a), and with the scatter and systematic differences between different spectral mass estimates (Gallazzi et al. 2005).

3. We calculate SDSS SFRs from the O II emission line fluxes using Equation (2), the same equation used for zCOSMOS galaxies. These SDSS SFRs are then additionally corrected using the aperture corrections given by Brinchmann et al. (2004), corrections which should work for $z>0.04$, as also demonstrated by Kewley et al. (2005).

4. We use the Sersic indices in the $g$ band from the New York University value-added galaxy catalog (NYU-VAGC), from Sersic fits described in Blanton et al. (2005).

5. We exclude AGNs using the [O III] $\lambda 5007 / \mathrm{H} \beta$ versus [N II] $\lambda 6584 / \mathrm{H} \alpha$ diagnostic diagram, excluding objects that satisfy Equation (1) of Kauffmann et al. (2003b).

This results in a sample of 55,230 SDSS galaxies at $0.04<$ $z<0.08$. Applying the same mass cut as applied to produce the two mass-complete zCOSMOS samples, we are left with 21,497 SDSS galaxies at $0.04<z<0.08$ with $\log M_{*}>10.4$, and 8904 SDSS galaxies at $0.04<z<0.08$ with $\log M_{*}>10.7$.

\section{RESULTS}

\subsection{Specific Star Formation Rates and Downsizing}

The SFR per unit stellar mass, the specific SFR, is an indicator of the galaxy star formation history, since 1 /SSFR defines a characteristic timescale of the stellar mass build-up. The inverse of the specific $\mathrm{SFR}, T_{\mathrm{SFR}}$, is the time required for the galaxy to form all of its stellar mass at the current SFR and this is shown on the right axis of Figure 4. The ages of the universe for the two respective redshift ranges considered are shown as the horizontal dashed magenta lines. Galaxies with $T_{\mathrm{SFR}}$ higher than the age of the Universe at that time are relatively "quiescent" and must have had a higher SFR in the past, while those with $T_{\mathrm{SFR}}$ less than the age of the Universe may be thought of as "active" or "forming," in the sense that they cannot have maintained this high SFR for all of their lifetime. If the SFR is constant or slowly declining, then "forming" galaxies will follow a roughly diagonal track in Figure 4 as they age toward lower redshifts, whereas "quiescent" galaxies will follow a nearly vertical path downwards toward lower SSFRs (the two models on the right in the panels of Figure 4).

It should be remembered that Figure 4 shows all galaxies in the zCOSMOS samples, regardless of whether they belong to the mass-complete samples to the right of the heavy vertical lines in the two panels. This figure shows two well known phenomena: first, the well known downsizing effect is observed in the sense that at $0.5<z<0.7$ very few galaxies with masses above $10^{10.8} M_{\odot}$ (to the right of the vertical, solid magenta line, in the yellow, hatched region) have SSFRs above the dashed magenta lines, while at $0.7<z<0.9$ there exist several dozens of galaxies above $10^{10.8} M_{\odot}$ that are "forming." Second, it is also obvious from the figure that galaxies with different Sersic indices have different star formation histories, even at a 


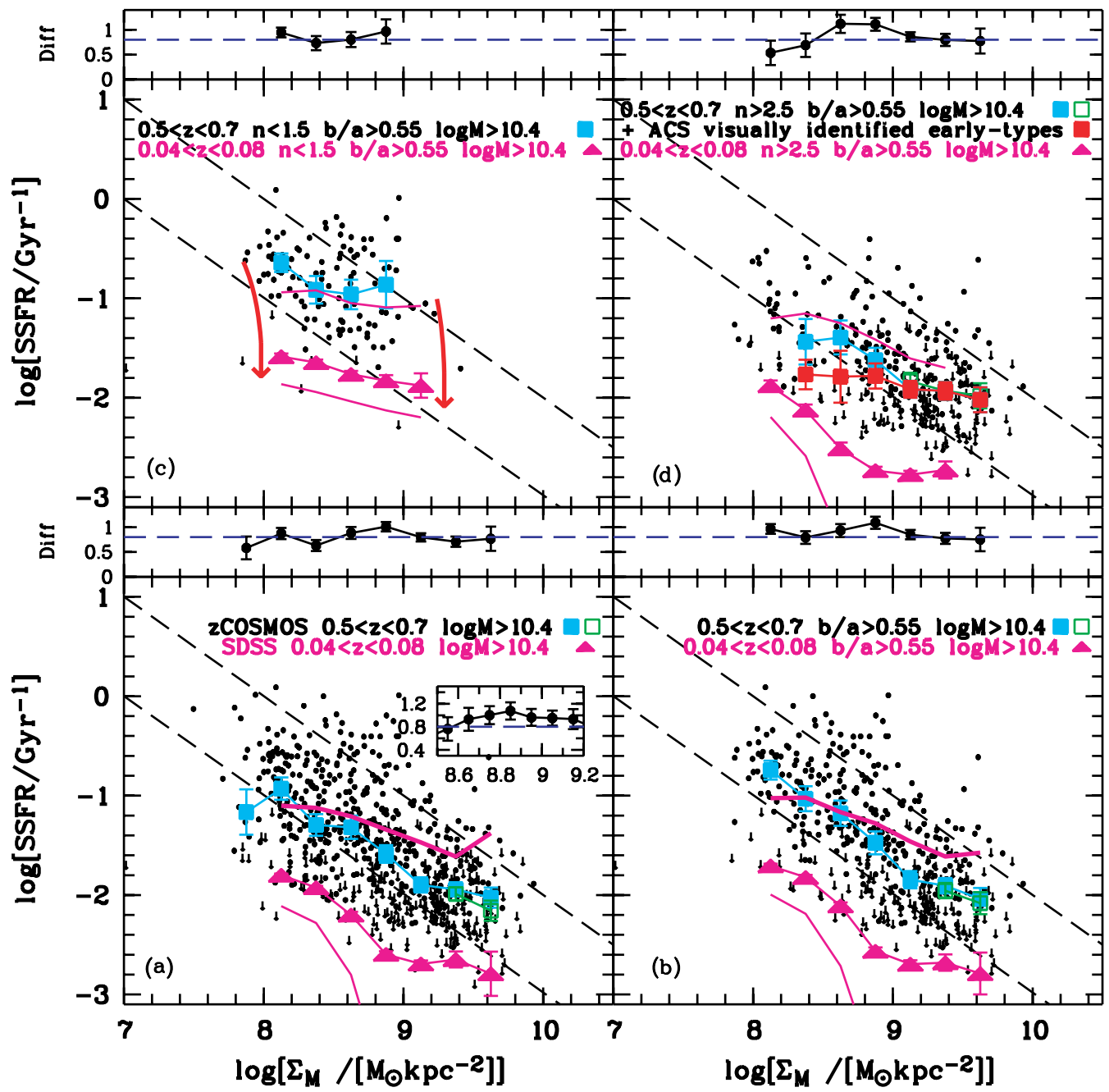

Figure 7. The SSFR vs. stellar surface mass density $\Sigma_{M}$ of the zCOSMOS mass-complete sample at $0.5<z<0.7$ (black dots), compared to the median and percentile curves summarizing the SDSS relations from Figure 5 (magenta points and lines). The diagonal dashed lines correspond to SFR surface densities $\Sigma_{\mathrm{SFR}}=0.01 M_{\odot} / \mathrm{yr} / \mathrm{kpc}^{2}$, and $0.1 M_{\odot} / \mathrm{yr} / \mathrm{kpc}^{2}$, respectively. The SSFR median values of zCOSMOS galaxies for different $\Sigma_{M}$ bins are shown as cyan filled squares, while for the highest $\Sigma_{M}$ we also show the mean SSFR values derived from coadded spectra as green open squares. Panel (a) shows all galaxies in the zCOSMOS mass-complete sample, panel (b) only galaxies with an axis ratio $b / a>0.55$, panel (c) galaxies with $b / a>0.55$ and $n<1.5$, and panel (d) galaxies with $b / a>0.55$ and $n>2.5$. The respective small panels above the main ones (and the insert in panel (a) with finer grid in $\Sigma_{M}$ ) show as filled squares (circles) the difference between the median zCOSMOS and SDSS SSFR values in a given $\Sigma_{M}$ bin. For $n<1.5$ (disk) galaxies the SSFR stays roughly constant with $\Sigma_{M}$, with the median SSFR at a given $\Sigma_{M}$ being about six times higher at $z \sim 0.6$ than in SDSS. Red arrows in panel (c) are model galaxy tracks discussed in Section 3.4. For $n>2.5$ (early-type) galaxies the SSFR declines with $\Sigma_{M}$ for both zCOSMOS and SDSS galaxies, but there is again a shift to higher SSFR with about the same factor of about five to six, and also a modestly higher $\Sigma_{M}$ at the higher redshift. Almost all the galaxies causing the upturn at low $\Sigma_{M}$ are clearly galaxies with disks, and the median SSFR of $n>2.5$ genuine early-type galaxies (see Section 3.3) is also almost independent of $\Sigma_{M}$ (red squares in panel (d)).

fixed mass: late-type galaxies with $n<2.5$ have mainly high SSFRs, which indicate continuing star formation, and possibly a recent onset of star formation, whereas early-type galaxies with $n>2.5$ have mostly low SSFR with $T_{\mathrm{SFR}}$ higher than the age of the Universe, indicating higher SFRs in the past and/or a higher redshift of their onset of star formation.

\subsection{The SDSS SSFR $-\Sigma_{M}$ Relation}

In Figure 5, we plot the SSFR versus $\Sigma_{M}$ diagram for the $0.04<z<0.08$ SDSS galaxies with $\log M_{*}>10.4-$ the sample that should be directly comparable to the $0.5<z<0.7$ zCOSMOS sample. Individual measurements are shown as green dots, the median SSFR values in different $\Sigma_{M}$ bins as magenta triangles, and, following Figure 9 of Kauffmann et al. (2006), 25th and 95th percentiles of the distribution of SSFR in the respective $\Sigma_{M}$ bin as solid magenta lines. The error bars shown are estimates of the error in the median, and are computed as $\Delta x=\left(x_{0.84}-x_{0.16}\right) / \sqrt{N}$, where $N$ is the number of galaxies in each $\Sigma_{M}$ bin, and $x_{0.84}$ and $x_{0.16}$ denote the 84th and 16th percentiles of the SSFR distribution. Panel (a) shows all galaxies, while panel (b) shows only the SDSS galaxies with an axis ratio $b / a>0.55$ (see 2.1.4 above). Panel (c) shows the low Sersic index $(n<1.5)$ objects with $b / a>0.55$, and panel (d) shows the $n>2.5$ SDSS galaxies, again with $b / a>0.55$.

In a previous study of the SSFR $-\Sigma_{M}$ relation for the SDSS sample, Kauffmann et al. (2006) found evidence that the distribution of star formation histories changes qualitatively above a characteristic surface mass density $\log \Sigma_{M \text { char }} \sim 8.5$. Moreover, for higher mass SDSS galaxies, Kauffmann et al. (2006) showed in their Figure 9 that the average SSFR remains constant below $\Sigma_{M \text { char }}$ and decreases at higher surface mass densities. This behavior is seen in panels (a) and (b) of Figure 5.

This trend is clearly due to the change over of different structural types from disk-dominated low Sersic index $(n<1.5)$ 


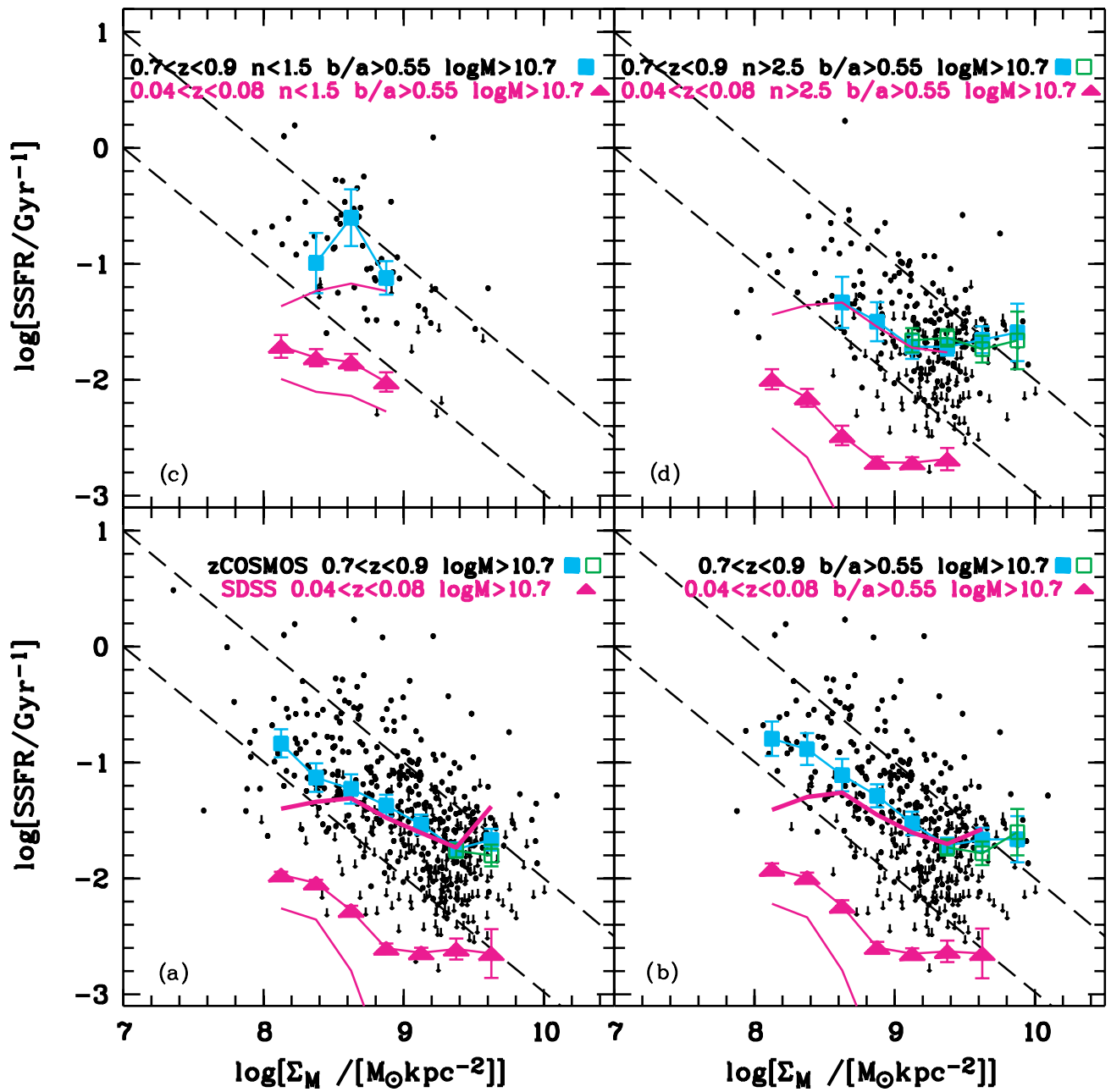

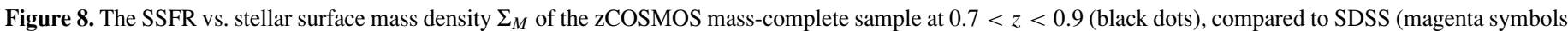

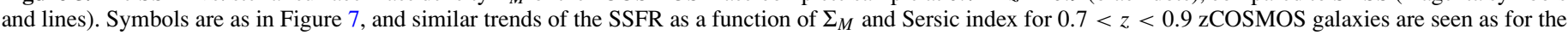
slightly lower redshift $0.5<z<0.7$ objects (Figure 7).

(A color version of this figure is available in the online journal.)

galaxies (in panel (c) of Figure 5) to bulge-dominated high Sersic index $(n>2.5)$ galaxies (panel (d) of Figure 5) as the $\Sigma_{M}$ increases. This change over is clearly seen in Figure 6, which shows the fraction of $n<1.5$ and $n>2.5$ SDSS galaxies (always with $b / a>0.55$ ) in different $\Sigma_{M}$ bins (magenta open and filled triangles). The fraction of $n>2.5$ SDSS objects shows a sharp increase at the point where the SSFR abruptly changes in Figure 5, i.e., at a transition surface mass density $\log \Sigma_{M \text { trans }} \sim 8.45$ (dashed magenta vertical line in Figure 6), close to the characteristic surface mass density $\log \Sigma_{M \text { char }} \sim 8.5$ of the break in the SSFR $-\Sigma_{M}$ relation.

Inspection of panel (c) of Figure 5 shows that the median SSFR for the late-type low Sersic SDSS galaxies $(n<1.5)$ and the plotted percentiles are all almost constant with $\Sigma_{M}$ - they decline very slightly with increasing $\Sigma_{M}$. In contrast, we see a rather steep drop in both the median and the 25th percentile of SSFR for the early-type $n>2.5$ SDSS galaxies (panel (d)), down to a SSFR level about 5-6 times below that of the latetype galaxies, where the bulk of the $n>2.5$ sample resides. We return to the form of the SSFR $-\Sigma_{M}$ for $n>2.5$ galaxies (panel (d)) below. In the meantime, we note that Figure 6 and panels (c) and (d) of Figure 5 explain how the shape of the median SDSS SSFR $-\Sigma_{M}$ relation for all galaxies is the result of the different SSFR $-\Sigma_{M}$ relations for $n<1.5$ and $n>2.5$ galaxies and the increasing fraction of early-type $n>2.5$ SDSS galaxies with increasing $\Sigma_{M}$ above $\Sigma_{M \text { trans }}$. The large difference in the median SSFR for early and late-type $(n>2.5$ and $n<1.5)$ galaxies "automatically" makes $\Sigma_{M \text { char }} \sim \Sigma_{M \text { trans }}$.

This explanation of the overall SSFR $-\Sigma_{M}$ relation should not be surprising but has not, as far as we are aware, been remarked upon previously, although Schiminovich et al. (2007) did note the different behavior of $n<2.5$ and $n>2.5$ SDSS galaxies.

\subsection{The zCOSMOS SSFR $-\Sigma_{M}$ Relation at $0.5<z<0.9$}

Figures 7 and 8 show the SSFR versus $\Sigma_{M}$ for zCOSMOS galaxies at $0.5<z<0.9$, and the comparison with the median and percentile curves summarizing the SDSS relations from Figure 5. The individual measurements for the zCOSMOS masscomplete sample at $0.5<z<0.7$ (Figure 7) and $0.7<z<0.9$ (Figure 8) are shown as black dots, and zCOSMOS median SSFR values for different $\Sigma_{M}$ bins as cyan, filled squares.

It should be noted that in SDSS the number of galaxies with no measurements (upper limits) of SSFR is always lower than $50 \%$ in all $\Sigma_{M}$ bins we are considering, so the median SSFR in the respective $\Sigma_{M}$ bin is always well defined. Unfortunately, this is not the case for the highest $\Sigma_{M}$ bins $\left(\log \Sigma_{M}>9\right)$ in the zCOSMOS sample: more than $50 \%$ of the galaxies in these bins 


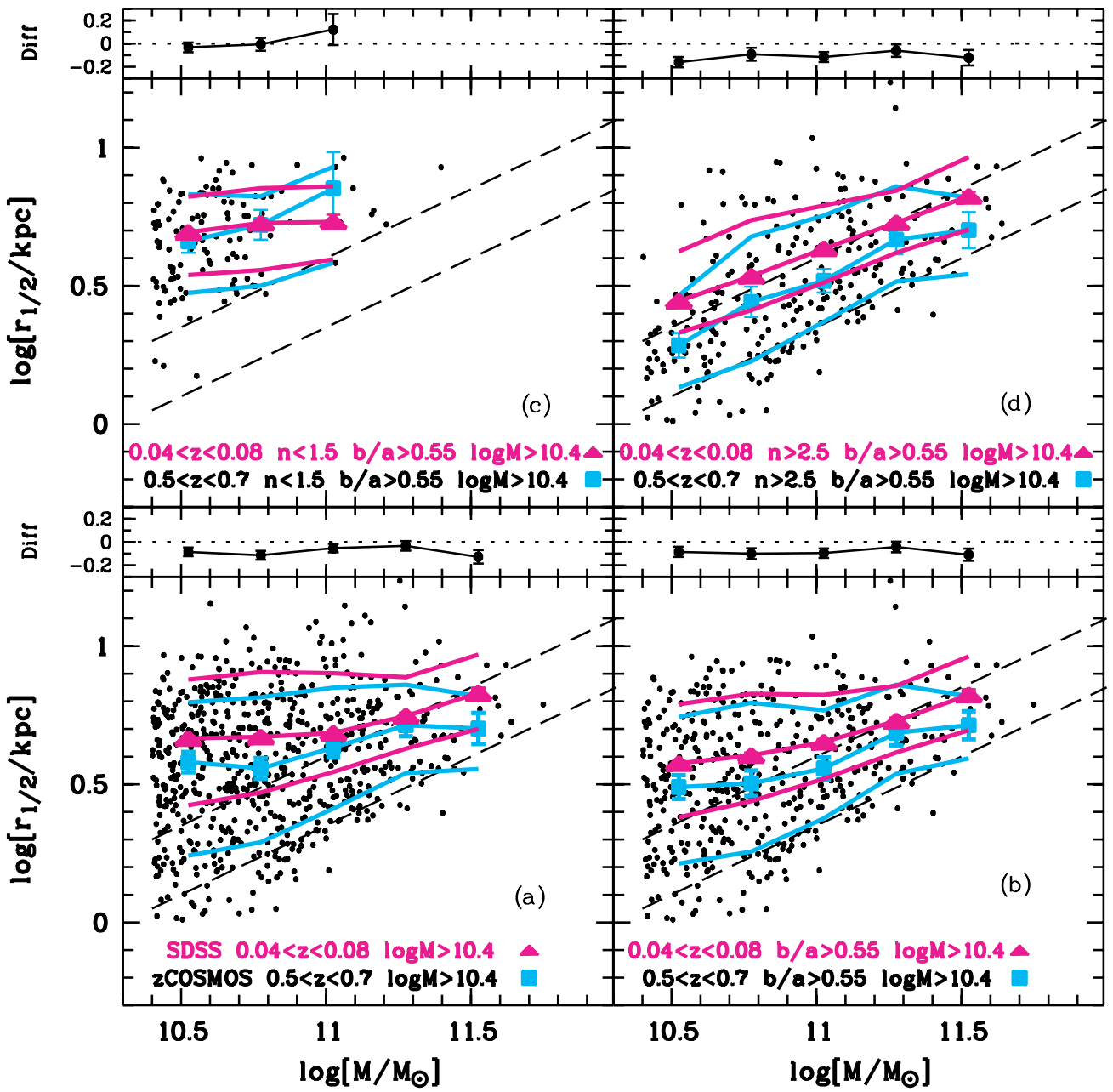

Figure 9. The semimajor half-light radius $r_{1 / 2}$ in the observed ACS $I$ band (corresponding roughly to rest-frame $B$ band for the redshift range $0.5<z<0.9$ ) versus stellar mass for the zCOSMOS mass-complete sample at $0.5<z<0.7$. Individual zCOSMOS measurements are shown as black dots, and median values as filled cyan squares. The median SDSS half-light radii in different mass bins derived from $g$-band images are shown as filled magenta triangles, while the 16th and 84th percentiles of $r_{1 / 2}$ of the SDSS and zCOSMOS galaxies in each mass bin are shown as solid magenta and cyan lines, respectively. The two dashed diagonal black lines show mass surface densities of $\log \Sigma_{M}=9$ (upper line), and $\log \Sigma_{\mathrm{M}}=9.5$, respectively. Panel (a) shows all galaxies in the mass-complete sample, panel (b) galaxies with an axis ratio $b / a>0.55$, panel (c) galaxies with $b / a>0.55$ and $n<1.5$, and panel galaxies with $b / a>0.55$ and $n>2.5$. The respective small panels above the main ones show as filled squares the difference between the median zCOSMOS and SDSS $r_{1 / 2}$ values in a given mass bin. For $n<1.5$ galaxies there is almost no evolution in the stellar mass-size relation between $z \sim 0.7$ and $z \sim 0$, while for $n>2.5$ objects the average half-light radius of galaxies at a given mass is smaller by $\sim 25 \%$ at $z \sim 0.7$

have only upper limits for the [O $\mathrm{II}]$ fluxes from Platefit_VIMOS. To overcome this problem, we first simply computed a median by setting all upper limits to actual values. However, we also generated an "average" coadded spectrum of all the spectra in each $\Sigma_{M}$ bin, and derived a mean SSFR from that, as follows. Each individual zCOSMOS spectrum (with a spectral resolution of $2.55 \AA$ ) was first transformed to its rest-frame and rebinned to a common grid of $2 \AA$ per pixel. These individual rest frame spectra were then normalized to the average value in a featureless region of the continuum and averaged. The [O II] flux from the coadded spectrum was transformed to a SFR using the usual calibration, and to a SSFR using an average mass of the galaxies in the respective $\Sigma_{M}$ bin. In fact, the mean SSFR value for the $\log \Sigma_{M}>9$ bins (shown as green open squares in Figures 7 and 8) derived in this way were found to be in good agreement with the median values that were obtained by setting the upper limits to their maximum values (cyan symbols).

For reasons mentioned in Section 2.1.4, we focus primarily on galaxies with $b / a>0.55$. Comparison of panels (a) and (b) of Figures 7 and 8 shows that this does not have a big effect on the analysis. The SSFR $-\Sigma_{M}$ relation in zCOSMOS has the same general shape as that for the SDSS across the whole range of $\Sigma_{M}$. However, the curve is shifted across the full range of $\Sigma_{M}$ to SSFR values that are about a factor of five to six higher in zCOSMOS than in SDSS. There is also a small shift of about 0.1-0.2 dex in the characteristic $\Sigma_{M \text { char }}$ toward higher surface mass densities at higher redshifts, indicated by a localised blip in the change in SSFR around $\Sigma_{M \text { char }}$. The shift in $\Sigma_{M \text { char }}$ is seen in the small panels of Figure 7 which show the difference between the median zCOSMOS and SDSS SSFR values in a given $\Sigma_{M}$ bin. If the shape of the SSFR $-\Sigma_{M}$ relation stays the same but there is a horizontal shift in $\Sigma_{M \text { char }}$, then the difference between the median zCOSMOS and SDSS SSFRs will be constant but with a localized excess in the region over which $\Sigma_{M \text { char }}$ has increased. This is seen as a blip in the small panels above panels (a), (b), and (d) of Figure 7. To illustrate more clearly the amount by which $\Sigma_{M \text { char }}$ shifts (0.1-0.2 dex) toward higher surface mass densities at higher redshifts, we have also included in Figure 7(a) 


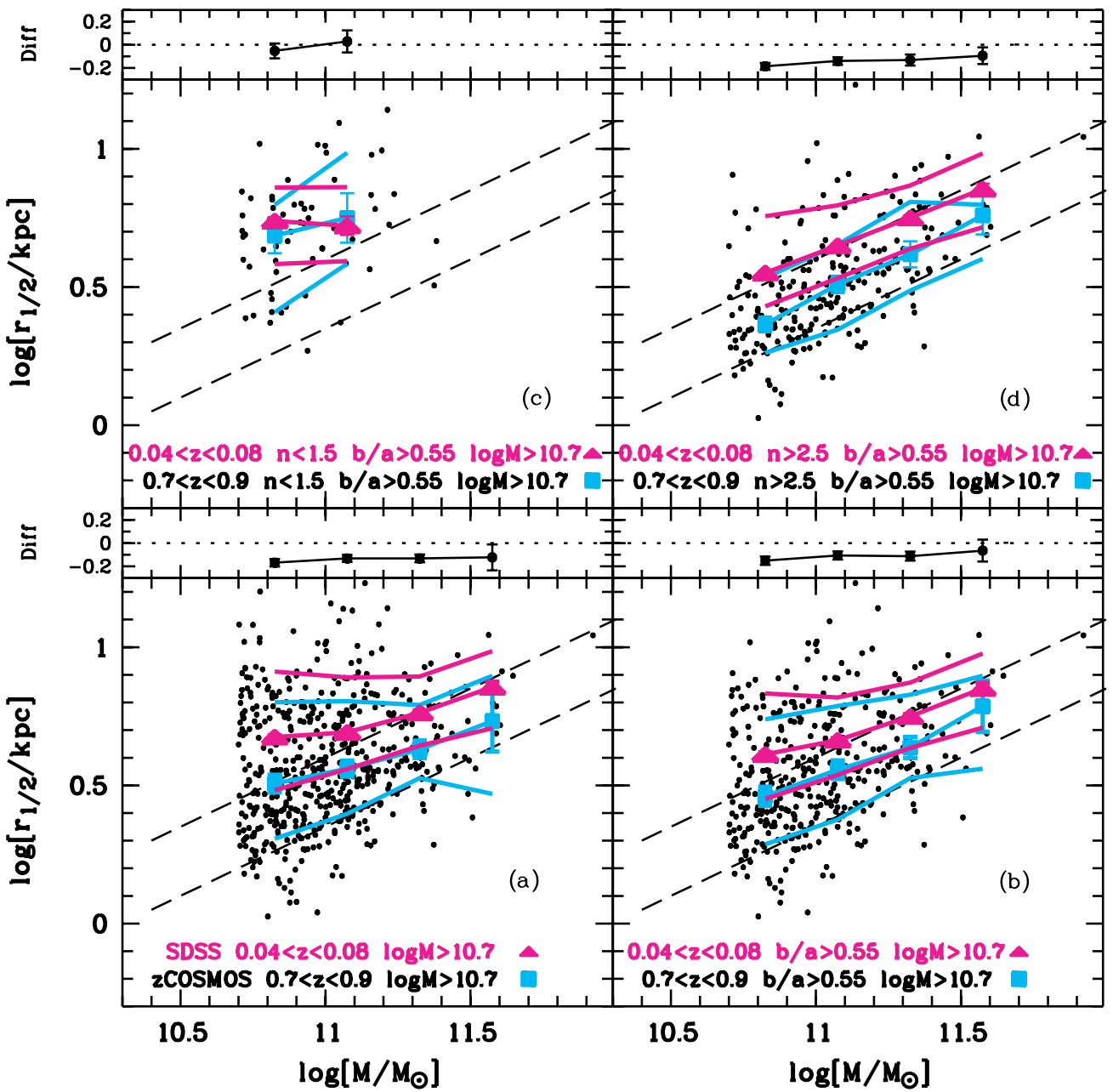

Figure 10. The semimajor half-light radius $r_{1 / 2}$ versus stellar mass for the zCOSMOS mass-complete sample at $0.7<z<0.9$ (black dots and cyan symbols and lines) compared to SDSS (magenta symbols and lines). Symbols are as in Figure 9, and similar trends of the stellar mass-size relation are seen here for $0.7<z<0.9$ zCOSMOS galaxies as seen in Figure 9 for the slightly lower redshift $0.5<z<0.7$ objects. However, the change in sizes of the early-type galaxies (panel (d)) becomes even more pronounced and this leads to a further increase in $\Sigma_{M \text { trans }}$ and $\Sigma_{M \text { char }}$.

(A color version of this figure is available in the online journal.)

an insert showing a part of the small panel above panel (a) with a finer grid in $\Sigma_{M}$.

The rise in median SSFR is seen both in the entire sample of zCOSMOS and SDSS galaxies (with or without the $b / a>0.55$ cut) and in the individual $n<1.5$ and $n>2.5$ subsets shown in panels (c) and (d) of the Figures 7 and 8. This rise in SSFR is essentially the same as the rise in the global SFRD in the Universe as a whole to this redshift (e.g., Lilly et al. 1996; Hippelein et al. 2003; Hopkins \& Beacom 2006, and references therein). The importance of this result is that it indicates that galaxies of all $\Sigma_{M}$ are contributing, proportionally, to this global evolution in the SFRD. Although the high $\Sigma_{M}$ galaxies with $\Sigma_{M}>\Sigma_{M \text { char }}$ (mostly early type galaxies with $n>2.5$ ) have a SSFR that is a factor of about six lower, on average, than the galaxies of the same mass with lower $\Sigma_{M}<\Sigma_{M \text { char }}$ (generally late type galaxies with $n<1.5$ ), the rise in SSFR by a factor of five to six is seen across the board.

As in SDSS, the SSFR for $n<1.5$ galaxies (panel (c) in Figures 7 and 8) remains roughly constant with $\Sigma_{M}$, albeit at a level that is elevated with respect to SDSS by about a factor of six. The shape of the relation for $n>2.5$ galaxies (panel (d) of Figures 7 and 8) is also similar to that seen in the SDSS, with a decline in the SSFR around $\Sigma_{M \text { char }}$ and a roughly constant SSFR at higher surface mass densities. The SSFR is again uniformly elevated across the range of $\Sigma_{M}$ by a factor of about five to six.

With the better resolution of the ACS COSMOS images we can now examine the galaxies which are causing the upturn in SSFR of $n>2.5$ galaxies at the lower $\Sigma_{M}$ in panel (d) of Figures 7 and 8. Most of the zCOSMOS galaxies with high SSFRs and lower $\Sigma_{M}$ that are responsible for this upturn are clearly disk galaxies with a dominant bulge. If we remove these from the sample, as indicated by the red points in the panel (d) of Figure 7, then we find a SSFR $-\Sigma_{M}$ relation that is essentially flat. We suspect that the same would also hold for the $n>2.5$ SDSS sample. Although we have had to parameterize the galaxy morphology in terms of a crude Sersic index, in order to achieve uniformity between SDSS and zCOSMOS, we would predict that the form of the SSFR $-\Sigma_{M}$ relation would consist of two essentially flat relations in SSFR $-\Sigma_{M}$ for disks and spheroids separately, which partly overlap in $\Sigma_{M}$ and which are offset in SSFR by a factor of five to six. Both components appear to increase in SSFR by the same amount to the redshifts probed by this study. 


\subsection{The SDSS and zCOSMOS Stellar Mass-Size Relations}

In order to better understand the small shift in $\Sigma_{M \text { char }}$ with redshift, we have looked at the relationship between mass and half-light radius in both Sersic subsamples at both SDSS and zCOSMOS redshifts. Figure 9 shows the semimajor half-light radius in the observed ACS $I$ band (corresponding roughly to rest-frame $B$ band for this redshift range) versus stellar mass for the zCOSMOS mass-complete sample at $0.5<z<0.7$. Black dots are individual zCOSMOS measurements, while cyan squares denote the median $r_{1 / 2}$ values in the respective mass bin. The SDSS median half-light radii in different mass bins derived from $g$-band images (as described in Section 2.2) are shown as filled, magenta triangles. The 16th and 84th percentiles of $r_{1 / 2}$ in the SDSS and zCOSMOS galaxies in each mass bin are shown as solid magenta and cyan lines, respectively. The two dashed black lines show constant mass surface densities of $\log \Sigma_{M}=9$ (upper line), and $\log \Sigma_{M}=9.5$, respectively. As before, panel (a) of Figure 9 shows all galaxies in the zCOSMOS mass sample, panel (b) galaxies with $b / a>0.55$, panel (c) galaxies with $b / a>0.55$ and $n<1.5$, and panel (d) galaxies with $b / a>0.55$ and $n>2.5$. The distribution of sizes of SDSS galaxies is displaced to larger sizes at a given mass compared with zCOSMOS when galaxies of all Sersic indices are considered (panels (a) and (b)). However, inspection of panels (c) and (d) of Figure 9 shows that this effect is almost entirely due to galaxies with $n>2.5$. The respective stellar mass-size relation for the slightly higher redshift $0.7<z<0.9$ galaxies is shown in Figure 10, and is discussed at the end of this section.

Panel (c) of Figure 9 shows that the stellar mass-size relation of $n<1.5$ zCOSMOS galaxies at $0.5<z<0.7$ does not differ significantly from the similar relation for SDSS $n<1.5$ galaxies. This is consistent with the GEMS result of Barden et al. (2005). These authors interpreted the almost negligible evolution with time in the stellar mass-size relation as support for an "inside-out" scenario. We would interpret an "insideout" scenario to involve a broadly constant surface mass density (whether measured at the center or at a characteristic half-mass radius) coupled with an increasing half-mass radius, i.e., an evolution parallel to the dashed lines in Figure 9.

However, as remarked above, the SFR in these galaxies declines rapidly (by a factor of about six as shown in panel (c) of Figure 7) since $z \sim 0.7$. The subsequent evolution of these $n<1.5$ zCOSMOS galaxies is described presumably by a declining SFR, as suggested by the paths of model galaxies shown in Figure 4 (solid green tracks). We show in panel (c) of Figure 7 the possible tracks of model galaxies (red solid arrows) with declining SFRs and with sizes assumed to be constant over this time period. The mass, and thus stellar mass density increases by $\Delta M_{*} \leqslant 0.3 M_{*}$. Given that the stellar mass-size relation seen in panel (c) of Figure 9 is almost flat, it is difficult to see much evidence for the required diagonal evolution in the size-mass plane. We therefore do not believe, over this redshift interval at least, that there is much evidence for inside out growth of disks. This is also consistent with the lack of evolution in the size function of disks (Lilly et al. 1998; Sargent et al. 2007).

In contrast, panel (d) in Figure 9 illustrates the difference in the stellar mass-size relation of high Sersic index $(n>2.5)$ zCOSMOS galaxies compared with the corresponding SDSS sample. Since the masses of early-type $n>2.5$ galaxies are not expected to evolve significantly between $z \sim 0.7$ and $z \sim 0$ because of the low and rapidly declining SSFRs (the two models

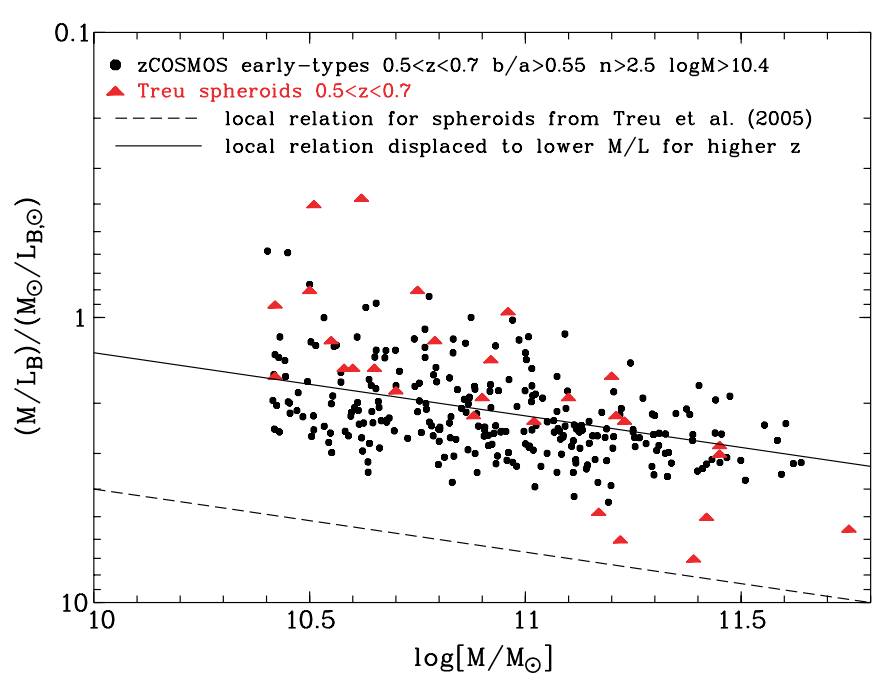

Figure 11. Comparison of the $B$-band $\mathrm{M} / \mathrm{L}$ ratios for zCOSMOS galaxies with $b / a>0.55$ and $n>2.5$ (black filled circles) with the M/L ratios obtained studying the fundamental plane for early-type (E+S0) galaxies (filled triangles) from Figure 15 of Treu et al. (2005), and the local relation (dashed line) for early-type (spheroid) galaxies taken from the same figure of Treu et al. (2005). The slope of the observed mean zCOSMOS M/L-mass relation agrees with the slope of the local relation, as indicated by the solid diagonal line, offset to lower $\mathrm{M} / \mathrm{L}$, as expected for a passively evolving population. Moreover, the spheroids from Treu et al. (2005) occupy a similar region of the $\mathrm{M} / \mathrm{L}-$ mass diagram as the zCOSMOS galaxies at $0.5<z<0.7$. This reassures that the derived stellar masses for $n>2.5$ zCOSMOS galaxies are reasonable, and further suggests that the changes in the stellar mass-size relation at $0.5<z<0.7$ in Figure 9 are due mainly to smaller sizes (or at least smaller measured $r_{1 / 2}$ ), at a given mass, in zCOSMOS compared with SDSS.

(A color version of this figure is available in the online journal.)

on the right in the left panel of Figure 4), we conclude that zCOSMOS objects are on average $25 \%$ smaller at a given mass (panel (d) in Figure 9). This change in size is similar to but smaller than found by Trujillo et al. (2007), who claimed $\sim 60 \%$ smaller $r_{1 / 2}$ on average compared to SDSS for objects at similar redshift with somewhat higher stellar masses $\left(\log M_{*}>11\right)$.

One might worry that stellar masses for $n>2.5 \mathrm{zCOSMOS}$ galaxies using Equation (1) are systematically wrong. To check the reliability of the calculated zCOSMOS masses, we compare the $B$-band $\mathrm{M} / \mathrm{Ls}$ of the zCOSMOS $n>2.5$ galaxies with the $\mathrm{M} / \mathrm{L}$ ratios obtained by studying the fundamental plane for early-type (E+S0) galaxies by Treu et al. (2005). Figure 11 shows the $B$-band $\mathrm{M} / \mathrm{L}$ ratios as a function of mass for our zCOSMOS sample (black filled circles), the M/L ratios of spheroids $(\mathrm{E}+\mathrm{S} 0)$ galaxies at $0.5<z<0.7$ from Figure 15 of Treu et al. (2005), and the local relation for early-type (spheroid) galaxies (dashed line) taken from their same figure. The slope of the mean observed zCOSMOS M/L-mass relation agrees with the slope of the local relation, as indicated by the solid diagonal line in Figure 11, which is obtained by shifting the local relation to the lower $\mathrm{M} / \mathrm{L}$ expected for a passively evolving population. Moreover, the $0.5<z<0.7$ spheroids from Treu et al. (2005) occupy a similar region of the M/Lmass diagram as the zCOSMOS galaxies at the same redshifts, suggesting that our stellar masses derived from optical colors for $n>2.5$ (early-type) galaxies are reasonable, and further suggesting that the changes in the stellar mass-size relation at $0.5<z<0.7$ in Figure 9 are mainly due to smaller sizes (or at least smaller measured $r_{1 / 2}$ ), at a given mass, in zCOSMOS compared with SDSS.

Similar results regarding the size evolution of early-type galaxies have been seen by Trujillo et al. (2007), with an even 


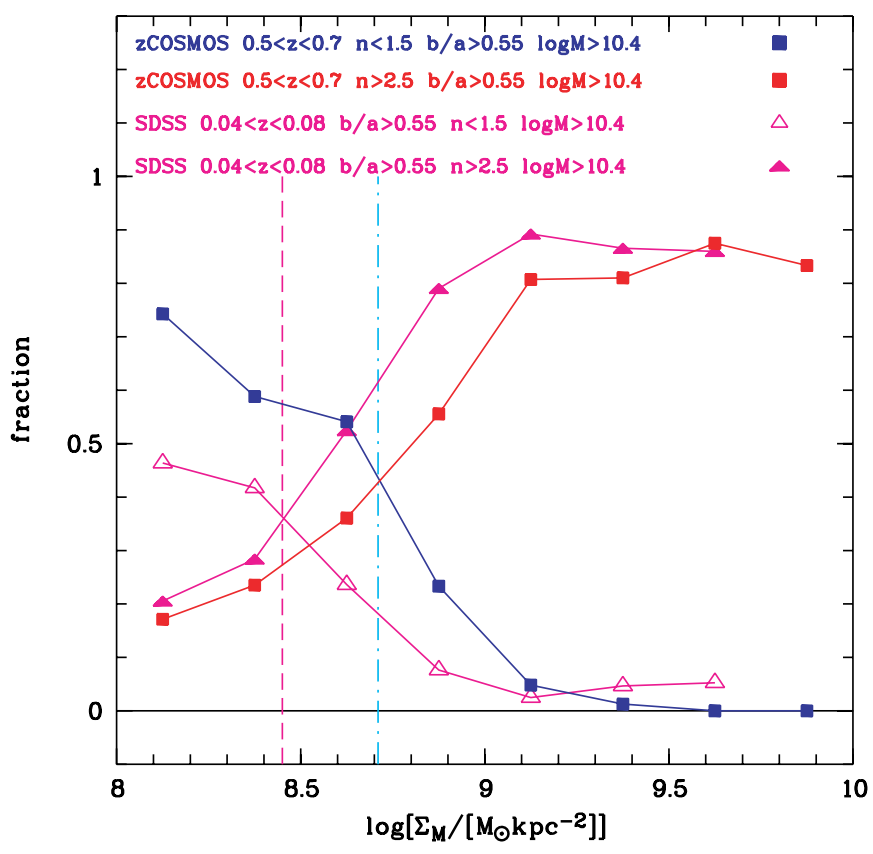

Figure 12. The fraction of $b / a>0.55$ objects with $n<1.5$ (blue filled squares) and $n>2.5$ (red filled squares) for zCOSMOS galaxies at $0.5<z<0.7$ from the mass-complete sample with $\log M_{*}>10.4$. The respective fractions for SDSS galaxies are shown as filled and open magenta triangles, for $n>2.5$ and $n<1.5$ galaxies, respectively (see also Figure 6 ). The transition surface mass density $\Sigma_{M \text { trans }}$ at which these curves cross is larger for zCOSMOS (dot-dashed cyan vertical line) than for SDSS galaxies (dashed magenta vertical line), and gives an explanation for the shift seen in the location of the step $\Sigma_{M \text { char }}$ in the median SSFR versus $\Sigma_{M}$ relation for zCOSMOS galaxies compared to that of the SDSS (Figures 7 and 8).

larger change in size, and at higher redshifts $z>1.4$ by Cimatti et al. (2008). Both these authors advocated dry mergers as a possible mechanism for the growth in size. Simulations with realistic boundary conditions by Naab et al. (2007) indicated that mergers could increase the size of galaxies while the mass changes only slightly. In the smooth envelope accretion scenario of Naab et al. (2007), accreted stars (mainly provided by minor mergers) form an envelope whose size increases smoothly with time. Khochfar \& Silk (2006) used a semianalytical model of galaxy formation to predict the redshift-size evolution of elliptical galaxies, and their Figure 4 shows that elliptical galaxies with masses above $10^{10} M_{\odot}$ could increase their sizes by a factor of about 1.25 from $z \sim 0.7$ to $z \sim 0$, consistent with our findings in panel (d) of Figure 9 for $n>2.5$ galaxies.

With these results in mind, we now return to understand the changing location of $\Sigma_{M \text { char }}$, the break in the SSFR $-\Sigma_{M}$ relation. Motivated by our SDSS analysis above, we show in Figure 12 the fraction of $n<1.5$ and $n>2.5$ Sersic index galaxies compared with the total number of galaxies (always with $b / a>0.55$ ) in different $\Sigma_{M}$ bins for zCOSMOS and SDSS. The cross-over point $\Sigma_{M \text { trans }}$ shifts to higher surface mass densities, presumably because, while the average $\Sigma_{M}$ of low Sersic index galaxies $(n<1.5)$ remains unaltered, that of the higher Sersic index galaxies $(n>2.5)$ shifts at higher redshifts to higher values, because of the apparent size evolution noted above. This shift in $\Sigma_{M \text { trans }}$ then produces the shift in $\Sigma_{M \text { char }}$ in the SSFR $-\Sigma_{M}$ relation.

The behavior of the SSFR $-\Sigma_{M}$ and stellar mass-size relations for $0.7<z<0.9$ zCOSMOS galaxies (Figures 8 and 10) in comparison with the equivalent SDSS samples (selected at higher masses because of the higher mass completeness limit in zCOSMOS, see Figure 4) is similar to that of galaxies at the slightly lower redshift of $0.5<z<0.7$, on which we have primarily focused in the foregoing. However, the change in sizes of the early-type galaxies becomes even more pronounced at $0.7<z<0.9$ and this leads to a further increase in $\Sigma_{M \text { trans }}$ and $\Sigma_{M \text { char }}$.

\section{SUMMARY AND CONCLUSIONS}

We have studied the relationships between SSFR and stellar mass density in two mass-complete samples of galaxies $\left(\log M_{*}>10.4\right.$ for $0.5<z<0.7$ and $\log M_{*}>10.7$ for $0.7<z<0.9$ ) drawn from the zCOSMOS survey, and the equivalent mass-complete samples of SDSS objects to draw the following conclusions:

1. The median SSFR of SDSS galaxies is almost independent of $\Sigma_{M}$ for low values of $\Sigma_{M}$, but then abruptly changes at a surface mass density $\Sigma_{M \text { char }} \sim 8.5$, in agreement with previous studies. This step function is clearly due to the change over of different structural types from disk-dominated low Sersic galaxies $(n<1.5)$ to bulgedominated high Sersic galaxies $(n>2.5)$ as the $\Sigma_{M}$ increases. The population mix changes over at a transition surface mass density $\log \Sigma_{M \text { trans }} \sim 8.45$, which is almost identical to the characteristic surface mass density at which the SSFR changes, $\Sigma_{M \text { char }}$, identified by Kauffmann et al. (2006).

2. The shape of the SSFR $-\Sigma_{M}$ relation in zCOSMOS at $0.5<z<0.9$ is very similar to that of the SDSS, with a roughly uniform increase in the average SSFR by a factor of five to six that is broadly independent of $\Sigma_{M}$ and which occurs in both early and late type galaxies with $n>2.5$ and $n<1.5$, respectively. There is also a small increase of $0.1-0.2$ dex in $\Sigma_{M \text { char }}$ at the higher redshifts.

3. The rise in SSFR is almost exactly the same as that seen in the overall SFRD of the Universe to this redshift, implying that galaxies across the full range of $\Sigma_{M}$, and with a wide range of Sersic index, are all contributing, proportionally, to the increase in the SFRD.

The modest increase in $\Sigma_{M \text { char }}$ by $\sim 0.1-0.2$ dex in zCOSMOS relative to the SDSS is naturally explained by differences in the size-mass relations for disk-dominated $(n<1.5)$ and bulge-dominated $(n>2.5)$ galaxies. Whereas the former have a size-mass relation that does not change with redshift, as also found by Barden et al. (2005), the latter are smaller at higher redshifts, as also seen in the samples of Trujillo et al. (2007), pushing individual galaxies to high $\Sigma_{M}$ values. This increases $\Sigma_{M \text { trans }}$, and thus $\Sigma_{M \text { char }}$, at the higher redshifts.

4. The median SSFR of disk-dominated galaxies $(n<1.5)$ is almost independent of surface mass density at both redshifts, but is about six times higher in zCOSMOS galaxies at $z \sim 0.7$ than in SDSS. With this strong decline in the average SSFR, the masses of the disk-dominated galaxies are unlikely to grow by more than $\sim 30 \%$ from $z \sim 0.7$ to $z \sim 0$. Coupled with the observed flat relation between size and stellar mass, this makes the evidence for "inside-out" growth of disks weak, at least over this redshift range (see Barden et al. 2005).

5. The upturn in the median SSFR for $n>2.5$ galaxies at lower $\Sigma_{M}$ is evidently due to galaxies with a significant disk component that have Sersic index $n>2.5$ due to a large dominant bulge. Their exclusion results in a median 

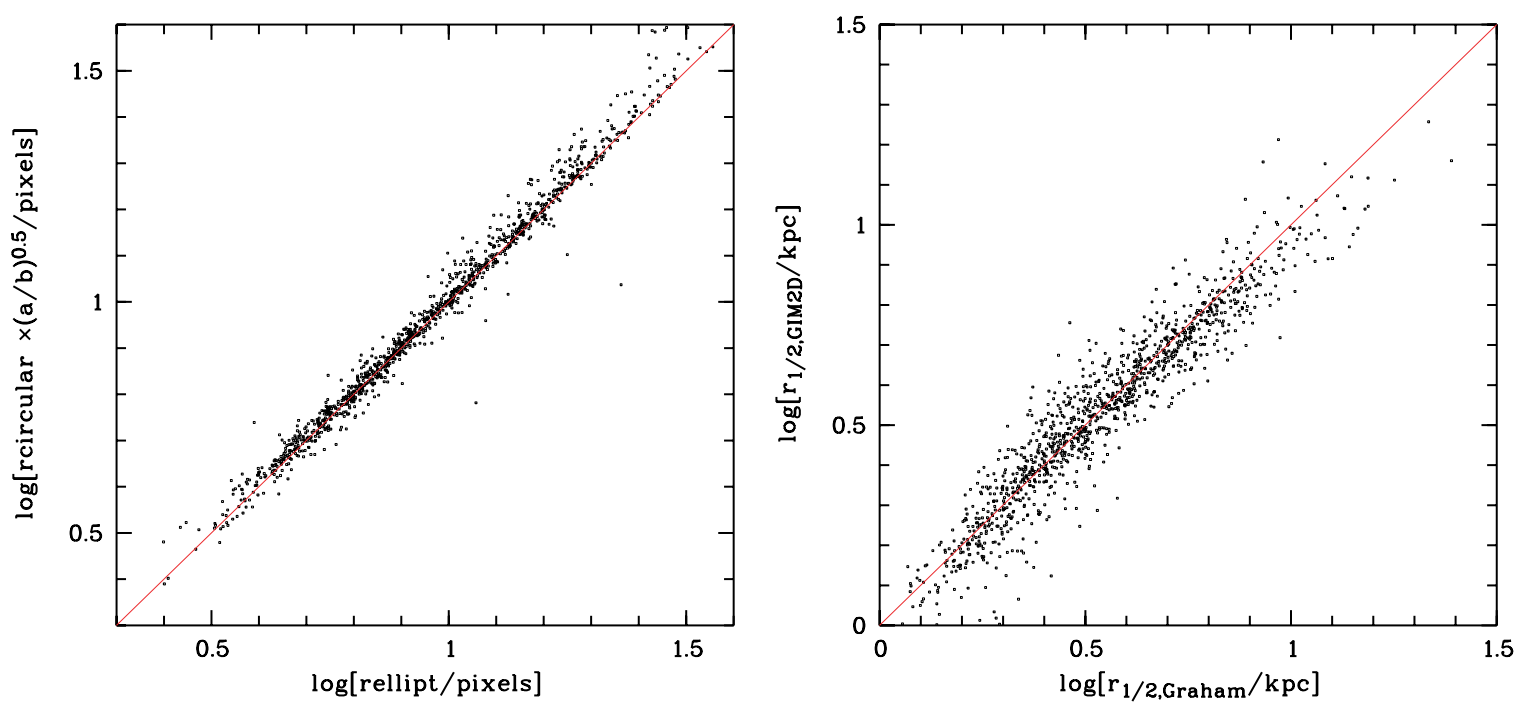

Figure 13. The left panel shows a good agreement (offset $0.009 \mathrm{dex}$, RMS 0.03 dex) between the COSMOS sizes (semimajor axis) computed directly from the ACS images using an elliptical aperture, and the sizes computed using circular apertures and multiplying them by $\sqrt{a / b}$. The right panel shows also a quite good agreement (offset 0.008 dex, RMS 0.07 dex) between the sizes (semimajor axis) computed with GIM2D, and the sizes computed starting from the circular Petrosian radius containing 50\% of the Petrosian flux and applying Equation (6) of Graham et al. (2005) and the $\sqrt{a / b}$ correction to compute the semimajor half-light radii (the same method as applied to SDSS and described in Section 2.2(1).

(A color version of this figure is available in the online journal.)

SSFR that is independent of $\Sigma_{M}$ also for the remaining $n>2.5 \mathrm{zCOSMOS}$ galaxies, but is about a factor of five to six lower than for the $n<1.5 \mathrm{zCOSMOS}$ galaxies.

Putting these together we arrive at the following main observation concerning the evolution of relatively massive galaxies since $z \sim 1$. In parallel with the evidence that, at all redshifts, the mean SSFR within a given population (either disk dominated with $n<1.5$ or bulge dominated with $n>2.5$ ) is independent of $\Sigma_{M}$, and that the observed SSFR $-\Sigma_{M}$ stepfunction relation is due, at all redshifts, to the changing mix of disk-dominated and bulge-dominated galaxies as $\Sigma_{M}$ increases and the strong difference in the average SSFR between disks and bulges, we find that the increase in SSFR with redshift is also independent of $\Sigma_{M}$ and also of Sersic index $n$. The increase matches that of the global SFRD of the Universe as a whole, suggesting that all types of galaxies are participating in the increase in SFR.

The conclusion is that the internal build-up of stellar mass in a galaxy is not strongly affected by $\Sigma_{M}$, beyond the overall structural role of $n$ (which clearly has a very large effect on the average SSFR, no doubt through the different structural components of disk and spheroid). The (relative) mean increase in SSFR back to $z \sim 1$ not only does not depend on $\Sigma_{M}$, it does not even seem to depend on $n$.

We would like to thank the anonymous referee for his or her suggestions and comments. We also want to thank M. Ciccolini and M. Aller for their help for testing the reliability of COSMOS size measurements. C.M. acknowledges support from the Swiss National Science Foundation. This work has been supported in part by grant ASI/COFIS/WP3110.

\section{APPENDIX}

\section{zCOSMOS VERSUS SDSS SIZE MEASUREMENTS}

It might be a concern that there are significant systematic differences between the size measurement methods for
zCOSMOS (as described in Section 2.1.3) and SDSS (as described in Section 2.2 (1)). To check the consistency of our size measurements for the two samples we used the ACS images of the mass-complete sample of $\sim 1200$ zCOSMOS galaxies at $0.5<z<0.9$ to test two issues: (1) are the sizes determined using a circular aperture (as in the case of SDSS) with a correction of $\sqrt{a / b}$ consistent with the sizes determined using elliptical apertures (as in GIM2D applied to zCOSMOS) and (2) are the GIM2D half-light radii consistent with a derivation of half-light radii starting from the Petrosian radius containing 50\% of the Petrosian flux and applying the corrections done for the SDSS sample described in Section 2.2(1). The results are shown in the two panels of Figure 13, and show that there are no significant systematic differences between the two size measurement methods in zCOSMOS and SDSS.

\section{REFERENCES}

Barden, M., et al. 2005, ApJ, 635, 959

Bauer, A. E., Drory, N., Hill, G. J., \& Feulner, G. 2005, ApJ, 621, 89

Blanton, M. R., et al. 2003a, ApJ, 594, 186

Blanton, M. R., et al. 2003b, AJ, 125, 2348

Blanton, M. R., et al. 2005, AJ, 129, 2578

Blanton, M. R., \& Roweis, S. 2007, AJ, 133, 734

Brinchmann, J., et al. 2004, MNRAS, 351, 1151

Brusa, M., et al. 2007, ApJS, 172, 353

Bruzual, G., \& Charlot, S. 2003, MNRAS, 344, 1000

Bundy, K., et al. 2006, ApJ, 651, 120

Capak, P., et al. 2007, ApJS, 172, 99

Caputi, K. I., et al. 2008, ApJ, 680, 939

Caputi, K. I., et al. 2009, ApJ, 691, 91

Chabrier, G. 2003, PASP, 115, 763

Cimatti, A., et al. 2008, A\&A, 482, 21

Feldmann, R., et al. 2006, MNRAS, 372, 565

Graham, A. W., et al. 2005, AJ, 130, 1535

Hippelein, H., et al. 2003, A\&A, 402, 65

Hopkins, A. M., \& Beacom, J. F. 2006, ApJ, 651, 142

Kauffmann, G., et al. 2003a, MNRAS, 341, 33

Kauffmann, G., et al. 2003b, MNRAS, 346, 1055

Kauffmann, G., et al. 2006, MNRAS, 367, 1394 
Kewley, L. J., Jansen, R. A., \& Geller, J. M. 2005, PASP, 117, 227

Khochfar, S., \& Silk, J. 2006, ApJ, 648, 21

Koekemoer, A. M., et al. 2007, ApJS, 172, 196

Lamareille, F., Mouhcine, M., Contini, T., Lewis, I., \& Maddox, S. 2004, MNRAS, 350, 396

Lin, L., et al. 2007, ApJ, 660, 51

Lilly, S. J., Le Fevre, O., Hammer, F., \& Crampton, D. 1996, ApJ, 460, L1

Lilly, S. J., et al. 1998, ApJ, 500, 75

Lilly, S. J., et al. 2007, ApJS, 172, 70

Maier, C., Lilly, S., Carollo, C. M., Stockton, A., \& Brodwin., M. 2005, ApJ, 634, 849

Maier, C., Lilly, S., Carollo, C. M., Meisenheimer, K., Hippelein, H., \& Stockton, A. 2006, ApJ, 639, 858

Maraston, C. 2005, MNRAS, 362, 799

Maller, A., Berlind, A. A., Blanton, M. R., \& Hogg, D. W. 2009, ApJ, 691, 394

Mignoli, M., et al. 2009, A\&A, 493, 39

Möllenhoff, C., Popescu, C. C., \& Tuffs, R. J. 2006, A\&A, 456, 941

Moustakas, J., Kennicutt, R. C., Jr, \& Tremonti, C. A. 2006, ApJ, 642, 775
Naab, T., Johansson, P. H., Ostriker, J. P. O., \& Efstathiou, G. 2007, ApJ, 658, 710

Noeske, K. G., et al. 2007a, ApJ, 660, 43

Noeske, K. G., et al. 2007b, ApJ, 660, 47

Pozzetti, L., et al. 2007, A\&A, 474, 443

Salim, S., et al. 2007, ApJS, 173, 267

Salpeter, E. E. 1955, ApJ, 121, 161

Sargent, M., et al. 2007, ApJS, 172, 434

Scarlata, C., et al. 2007, ApJS, 172, 406

Schiminovich, D., et al. 2007, ApJS, 173, 315

Scoville, N., et al. 2007a, ApJS, 172, 1

Scoville, N., et al. 2007b, ApJS, 172, 38

Silverman, J. D., et al. 2008, arXiv:0810.3653

Taniguchi, Y., et al. 2007, ApJS, 172, 9

Treu, T., et al. 2005, ApJ, 633, 174

Trujillo, I., et al. 2007, MNRAS, 382, 109

Willmer, C. N. A., et al. 2006, ApJ, 647, 853

Zamojski, M. A., et al. 2007, ApJS, 172, 468 